\title{
Interference Exploitation Precoding Made Practical: Optimal Closed-Form Solutions for PSK Modulations
}

\author{
Ang Li, Member, IEEE, and Christos Masouros, Senior Member, IEEE
}

\begin{abstract}
In this paper, we propose closed-form precoding schemes with optimal performance for constructive interference (CI) exploitation in the multiuser multiple-input single-output (MU-MISO) downlink, where the cases of both strict and nonstrict phase rotation are considered. For optimization with strict phase rotation, we mathematically derive the optimal precoding structure with Lagrangian and Karush-Kuhn-Tucker (KKT) conditions. By formulating its dual problem, the optimization problem is further shown to be equivalent to a quadratic programming (QP) over a simplex, which can be solved more efficiently. We then extend our analysis to the case of nonstrict phase rotation, where it is mathematically shown that a $K$-dimensional optimization for non-strict phase rotation is equivalent to a $2 K$-dimensional optimization for strict phase rotation in terms of the problem formulation. The connection with the conventional zero-forcing (ZF) precoding is also discussed. Based on the above analysis, we further propose an iterative closed-form scheme to obtain the optimal precoding matrix, where within each iteration a closed-form solution can be obtained. Numerical results validate our analysis and the optimality of the proposed iterative closed-form algorithm, and further show that the proposed iterative closed-form scheme offers a flexible performance-complexity tradeoff by limiting the maximum number of iterations, which motivates the use of CI precoding in practical wireless systems.
\end{abstract}

Index Terms-MIMO, precoding, constructive interference, optimization, Lagrangian, closed-form solutions.

\section{INTRODUCTION}

$\mathbf{M}$ ULTIPLE-input multiple-output (MIMO) systems have been widely acknowledged as a promising technology in the field of wireless communications, due to the significant gains over single-antenna systems [1]. When the channel knowledge is known at the base station (BS), the capacityachieving dirty-paper coding (DPC) scheme is proposed in [2] by pre-subtracting the interference prior to transmission. However, DPC is difficult to implement in practical systems due to the impractical assumption of infinite alphabet and its

Manuscript received December 17, 2017; revised May 18, 2018, and July 25, 2018; accepted September 04, 2018. The associate editor coordinating the review of this paper and approving it for publication was Dr. Mohammad Reza Nakhai. (Corresponding author: Ang Li.)

A. Li was with the Department of Electronic and Electrical Engineering, University College London, Torrington Place, London, WC1E 7JE, UK, and is now with the School of Electrical and Information Engineering, University of Sydney, Maze Crescent, Darlington NSW 2006, Australia. C. Masouros is with the Department of Electronic and Electrical Engineering, University College London, Torrington Place, London, WC1E 7JE, UK (email: ang.li.14@ucl.ac.uk, chris.masouros@ieee.org).

This work was supported by the Royal Academy of Engineering, UK, the Engineering and Physical Sciences Research Council (EPSRC) project EP/M014150/1 and the China Scholarship Council (CSC). high computational cost. To achieve a compromise between performance and complexity, its non-linear counterparts in the form of Tomlinson-Harashima precoding (THP) [3] and vector perturbation (VP) [4] have been proposed, which however are still too complicated for practice due to the inclusion of the sophisticated sphere-search algorithms. Therefore, lowcomplexity linear precoding schemes based on zero-forcing (ZF) have received increasing research attention [5], and a regularized $\mathrm{ZF}$ (RZF) scheme is proposed in [6] to further improve the performance of ZF. On the other hand, downlink precoding schemes based on optimization have also been a popular research topic [7]-[13]. Among the optimization-based schemes, one form of the optimization known as signal-tointerference-plus-noise ratio (SINR) balancing is to maximize the minimum SINR subject to a total power constraint [7], [8] or a per-antenna power constraint [9]. An alternative downlink precoding strategy targets at minimizing the total transmit power at the BS subject to a minimum SINR requirement [10]-[12]. It has been shown that the power minimization problems can be formulated either as a virtual uplink problem with power control or as a semi-definite programming (SDP) and solved via semi-definite relaxation (SDR) [11]. As for the SINR balancing problem, it is proven to be an inverse problem to the power minimization optimization, based on which schemes via bisection search [7] and iterative algorithms [10] have been proposed.

Nevertheless, both the closed-form and optimization-based precoding designs mentioned above have ignored the fact that interference can be beneficial and further exploited on on a symbol level [14], [15]. The concept of constructive interference (CI) was firstly introduced in [16], where it is shown that the instantaneous interference can be categorized into constructive and destructive. A modified $\mathrm{ZF}$ precoding scheme is then proposed in [17], where the constructive interference is exploited while the destructive interference is cancelled. A correlation rotation scheme has been further proposed in [18], where it is shown that the destructive interference can be manipulated and rotated such that all the interference becomes constructive. Subsequently, symbol-level precoding schemes based on convex optimization for CI has been proposed in [19], [20], where the concept of constructive region is introduced to relax the strict phase rotation constraint in [18] and achieve an improved performance. Further studies on the optimization-based CI precoding methods can be found in [20]-[23]. Due to the performance benefits over conventional schemes, the concept of CI has been extended 
to many wireless application scenarios, including cognitive radio [24]-[26], relay [27], vector perturbation [28], radar and cellular coexistence [29], wireless information and power transfer [30], mutual coupling exploitation [31], non-linear channel [32] and directional modulation [33]-[35]. Moreover, for massive MIMO regime which has become a hot research topic recently, while the ZF precoding is shown to be optimal for ideal fully-digital massive MIMO systems, the CI-based formulation is still useful for practical hardware-constrained massive MIMO systems, for example the constant envelope precoding in [36] and the 1-bit massive MIMO in [37], [38]. The above studies show that MIMO systems can benefit from the CI with a symbol-level precoding. Nevertheless, while the performance of CI-based precoding approaches is superior, they need to solve a convex optimization problem, which can be computationally inefficient, especially when executed on a symbol-by-symbol basis.

In this paper, we design low-complexity optimal and suboptimal solutions for CI precoding, culminating in closedform iterative precoders. We focus on CI exploitation for PSK modulations, as PSK modulations lead to a generic CI constraint formulation, which will be shown in the following. We consider an optimization problem where we maximize the distance between the constructive region and the detection thresholds such that the effect of CI is maximized. We firstly consider the optimization for strict phase rotation, where the phases of the interfering signals are rotated such that they are strictly aligned to the symbol of interest. By analyzing the formulated second-order cone programming (SOCP) optimization with Lagrangian and KKT conditions, we derive the structure of the optimal precoding matrix, which leads to an equivalent optimization and further simplifies the precoding design. By formulating the dual problem of the equivalent optimization problem, it is mathematically shown that the optimization for CI precoding is equivalent to a quadratic programming $(\mathrm{QP})$ optimization over a simplex, which finally leads to a closedform expression. We extend our analysis to the case of nonstrict phase rotation, where the phases of the interfering signals are rotated such that the resulting interfered signal is located within the constructive region. By following a similar approach for the case of strict phase rotation, we analytically show that the optimal precoding matrix for theses two scenarios shares a similar closed-form expression, and a $K$-dimensional optimization for non-strict phase rotation is equivalent to a $2 K$-dimensional optimization for strict phase rotation in terms of the problem formulation. Our above analysis also provides some insights on the connection between the CI precoding and the generic ZF precoding.

Moreover, our efforts to facilitate the symbol-level CI precoding in practice culminate in an iterative closed-form scheme to efficiently obtain the optimal precoding matrix, where a closed-form solution is obtained within each iteration. Numerical results validate our above analysis and the optimality of the proposed iterative closed-form method for both strict and non-strict phase rotation. Moreover, it is numerically shown that with only in a few iterations, the iterative closedform algorithm obtains optimal performance. By constraining the maximum number of iterations, we further obtain a flexible performance-complexity tradeoff for the proposed iterative method, based on its connection with conventional ZF precoding. Both of the above motivate the use of CI-based precoding in practical wireless systems.

For reasons of clarity, we summarize the contributions of this paper as:

1) We formulate the optimization problem for CI-based precoding, where we maximize the distance between the constructive region and the detection thresholds. We derive the optimal precoding matrix for strict phase rotation and further formulate an equivalent and simplified optimization problem.

2) The optimization for strict phase rotation is transformed and further shown to be equivalent to a QP problem over a simplex, which can be more efficiently solved than the originally formulated problem.

3) We extend our analysis to the case of non-strict phase rotation, where the expression of the optimal precoding structure is similar to the case of strict phase rotation. It is further shown that a $K$-dimensional optimization for nonstrict phase rotation is equivalent to a $2 K$-dimensional optimization for strict phase rotation in terms of the problem formulation.

4) We analytically study the connection between the CIbased and the generic ZF precoding, where it is shown that $\mathrm{ZF}$ precoding can be regarded as a special case of CI-based precoding with all the dual variables being zero.

5) We further propose an iterative closed-form algorithm to obtain the optimal precoding matrix for both the strict and non-strict phase rotation cases, where within each iteration a closed-form solution can be derived. We show that the closed-form precoder obtains the optimal performance in only a few iterations.

The remainder of this paper is organized as follows. Section II introduces the system model and briefly reviews CI. Section III includes the analysis for the optimization problems with both strict and non-strict phase rotation constraints. The connection between the CI precoding and conventional ZF precoding is discussed in Section IV, and the proposed iterative closed-form scheme is introduced in Section V. The computational costs of the optimization-based approach and the iterative algorithm are both discussed in Section VI. The numerical results are shown in Section VII, and Section VIII concludes the paper.

Notations: $a$, a, and A denote scalar, vector and matrix, respectively. $(\cdot)^{*},(\cdot)^{T},(\cdot)^{H}$ and $\operatorname{tr}\{\cdot\}$ denote conjugate, transposition, conjugate transposition and trace of a matrix, respectively. $j$ denotes the imaginary unit, and $\operatorname{vec}(\cdot)$ denotes the vectorization operation. $\mathbf{A}(k, i)$ denotes the entry in the $k$-row and $i$-th column of $\mathbf{A}$. $|\cdot|$ denotes the absolute value of a real number or the modulus of a complex number, and $\|\cdot\|_{2}$ denotes the 12-norm. $\mathcal{C}^{n \times n}$ represents an $n \times n$ matrix in the complex set, and $\mathbf{I}$ denotes the identity matrix. $\Re(\cdot)$ and $\Im(\cdot)$ denote the real and imaginary part of a complex number, respectively. $\operatorname{card}(\cdot)$ denotes the cardinality of a set. 


\section{System Model AND CONSTRUCTIVE InTERFERENCE}

In this section, the system model that we consider is firstly introduced, followed by a brief review of CI and the constructive region.

\section{A. System Model}

We consider a multiuser MISO system in the downlink, where the BS structure with a symbol-level precoding is depicted in Fig. 1. The iterative closed-form algorithm will be introduced in Section V. The BS with $N_{t}$ transmit antennas is simultaneously communicating with $K$ single-antenna users in the same time-frequency resource, where $K \leq N_{t}$. We focus on the downlink precoding designs and perfect CSI is assumed throughout the paper. The data symbol vector is assumed to be from a normalized PSK modulation constellation [19], denoted as $\mathbf{s} \in \mathcal{C}^{K \times 1}$. Then, the received signal at the $k$-th user can be expressed as

$$
r_{k}=\mathbf{h}_{k} \mathbf{W} \mathbf{s}+n_{k},
$$

where $\mathbf{h}_{k} \in \mathcal{C}^{1 \times N_{t}}$ denotes the flat-fading Rayleigh channel vector from user $k$ to the BS, and each entry in $\mathbf{h}_{k}$ follows a standard complex Gaussian distribution. $\mathbf{W} \in \mathcal{C}^{N_{t} \times K}$ is the precoding matrix and $n_{k}$ is the additive Gaussian noise with zero mean and variance $\sigma^{2}$ at the receiver.

\section{B. Constructive Interference}

$\mathrm{CI}$ is defined as the interference that pushes the received signals away from the detection thresholds [14]-[18]. CI for strict phase rotation refers to the cases where the phases of the interfering signals are controlled and rotated, such that they are strictly aligned to those of the data symbols of interest [18]. The constructive region has been further introduced in [19], where it is shown that the phases of the interfering signals may not be necessarily strictly aligned to that of the data symbols of interest, known as the non-strict phase rotation. It is demonstrated that, as long as the resulting interfered signals are located in the constructive region, this increases the distance to the detection thresholds and returns an improved performance. To show this intuitively, in Fig. 2 and Fig. 3 we depict the case for strict phase rotation and non-strict phase rotation respectively, where the constellation point $\left(\frac{1}{\sqrt{2}}+\frac{1}{\sqrt{2}} \cdot j\right)$ from a normalized QPSK constellation is employed as the example to illustrate these two cases. We can observe that for both strict phase rotation and nonstrict phase rotation, the distance of the received signals to the detection thresholds is increased, which will improve the detection performance.

\section{Constructive Interference Precoding}

In this section, we firstly focus on the CI precoding for strict phase rotation, and we further extend our analysis to the case of non-strict phase rotation.

\section{A. Strict Phase Rotation}

Before formulating the optimization problem, based on the geometry of the modulation constellation we firstly construct the conditions that the precoder should satisfy to achieve the strict phase rotation. In Fig. 2, without loss of generality we denote $\overrightarrow{O A}=t \cdot s_{k}$ and $t=|\overrightarrow{O A}|$ is the object to be maximized. We further assume that the node ' $\mathrm{B}$ ' denotes the noiseless received signal for user $k$ that is co-linear to $\overrightarrow{O A}$ for strict phase rotation, which leads to

$$
\overrightarrow{O B}=\mathbf{h}_{k} \mathbf{W} \mathbf{s} \text {. }
$$

Then, by introducing a real-valued scaling factor $\lambda_{k}$, we further express $\overrightarrow{O B}$ as

$$
\overrightarrow{O B}=\mathbf{h}_{k} \mathbf{W} \mathbf{s}=\lambda_{k} s_{k},
$$

where based on the geometry we can obtain that $\lambda_{k}$ is a real number, and the condition on $\lambda_{k}$ to achieve CI for strict phase rotation is given by

$$
\lambda_{k} \geq t, \forall k \in \mathcal{K}
$$

where $\mathcal{K}=\{1,2, \cdots, K\}$. With the above formulation, we can construct the optimization problem for strict phase rotation as

$$
\begin{array}{ll}
\mathcal{P}_{1}: & \max _{\mathbf{W}, t} t \\
\text { s.t. } & \mathbf{h}_{k} \mathbf{W} \mathbf{s}=\lambda_{k} s_{k}, \forall k \in \mathcal{K} \\
& \lambda_{k} \geq t, \forall k \in \mathcal{K} \\
& \|\mathbf{W} \mathbf{s}\|_{2}^{2} \leq p_{0}
\end{array}
$$

where $p_{0}$ denotes the total available transmit power. A symbollevel power constraint is employed, as the exploitation of CI is related to the transmit symbol vector, which will also be shown mathematically in the following. $\mathcal{P}_{1}$ belongs to the SOCP and can be solved with convex optimization tools such as CVX [19]. We decompose the precoding matrix into vectors

$$
\mathbf{W}=\left[\mathbf{w}_{1}, \mathbf{w}_{2}, \cdots, \mathbf{w}_{K}\right],
$$

and based on the virtual multicast formulation in [19] we obtain that each $\mathbf{w}_{i} s_{i}$ is identical. Accordingly, we obtain $\|\mathbf{W} \mathbf{s}\|_{2}^{2}=K^{2} \cdot\left\|\mathbf{w}_{i} s_{i}\right\|_{2}^{2}$ and $\sum_{i=1}^{K} s_{i}^{*} \mathbf{w}_{i}^{H} \mathbf{w}_{i} s_{i}=K \cdot\left\|\mathbf{w}_{i} s_{i}\right\|_{2}^{2}$, which leads to the equivalent transformation of the power constraint, given by

$$
\|\mathbf{W} \mathbf{s}\|_{2}^{2} \leq p_{0} \Rightarrow \sum_{i=1}^{K} s_{i}^{*} \mathbf{w}_{i}^{H} \mathbf{w}_{i} s_{i} \leq \frac{p_{0}}{K} .
$$

We further transform $\mathcal{P}_{1}$ in (5) into a standard minimization problem, expressed as

$$
\begin{array}{ll}
\mathcal{P}_{2}: & \min _{\mathbf{w}_{i}, t}-t \\
\text { s.t. } & \mathbf{h}_{k} \sum_{i=1}^{K} \mathbf{w}_{i} s_{i}-\lambda_{k} s_{k}=0, \forall k \in \mathcal{K} \\
& t-\lambda_{k} \leq 0, \forall k \in \mathcal{K} \\
& \sum_{i=1}^{K} s_{i}^{*} \mathbf{w}_{i}^{H} \mathbf{w}_{i} s_{i}-\frac{p_{0}}{K} \leq 0
\end{array}
$$




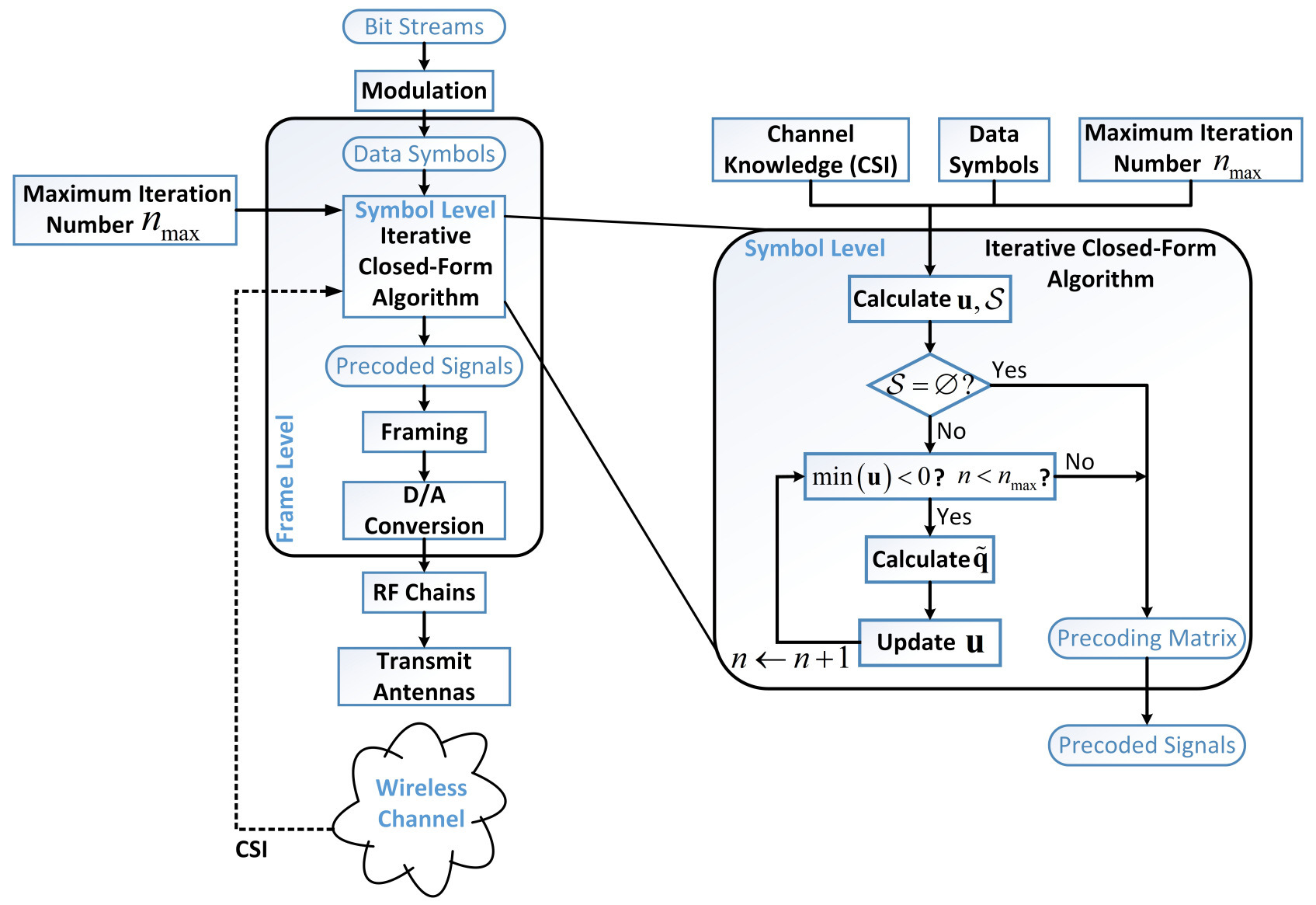

Fig. 1: A block diagram for the proposed symbol-level precoding based on CI

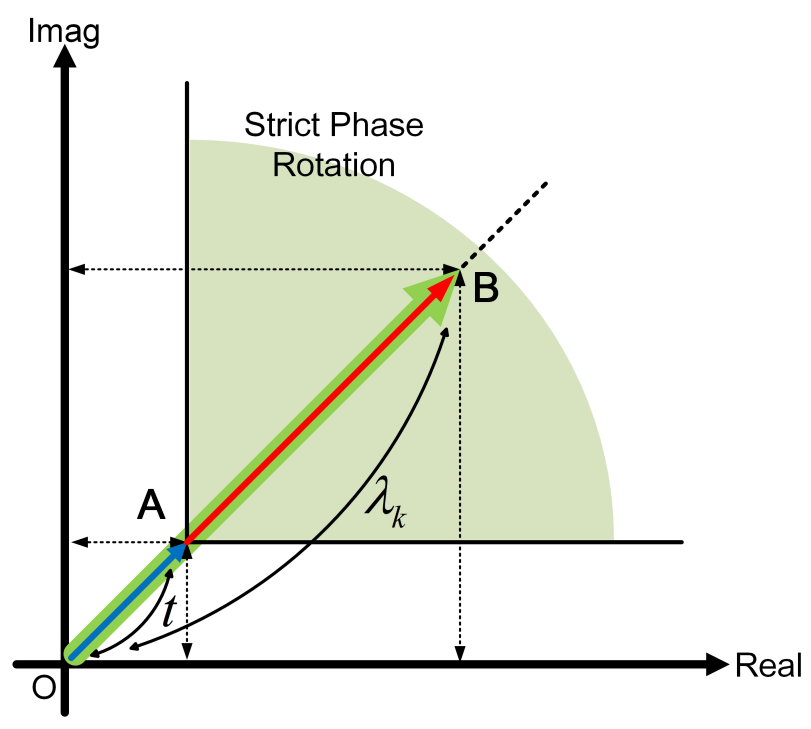

Fig. 2: Constructive interference, QPSK, strict phase rotation

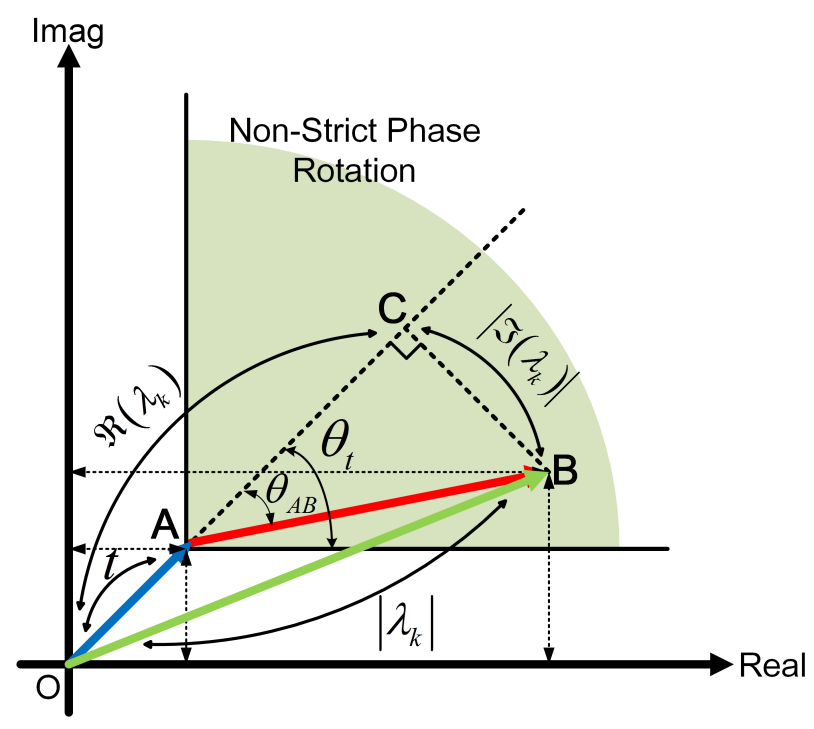

Fig. 3: Constructive region, QPSK, non-strict phase rotation

In the following we analyze $\mathcal{P}_{2}$ with Lagrangian and KKT conditions. The Lagrangian of $\mathcal{P}_{2}$ is expressed as [39]

$$
\begin{aligned}
& \mathcal{L}\left(\mathbf{w}_{i}, t, \delta_{k}, \mu_{k}, \mu_{0}\right)=-t+\sum_{k=1}^{K} \delta_{k}\left(\mathbf{h}_{k} \sum_{i=1}^{K} \mathbf{w}_{i} s_{i}-\lambda_{k} s_{k}\right) \\
& +\sum_{k=1}^{K} \mu_{k}\left(t-\lambda_{k}\right)+\mu_{0}\left(\sum_{i=1}^{K} s_{i}^{*} \mathbf{w}_{i}^{H} \mathbf{w}_{i} s_{i}-\frac{p_{0}}{K}\right),
\end{aligned}
$$

where $\delta_{k}, \mu_{k}$ and $\mu_{0}$ are the dual variables, and we have $\mu_{0} \geq$ 0 and $\mu_{k} \geq 0, \forall k \in \mathcal{K}$. Each $\delta_{k}$ may be complex as it is the dual variable with respect to the equality constraint. Based on the Lagrangian in (9), the KKT conditions for optimality can 
be obtained as

$$
\begin{array}{r}
\frac{\partial \mathcal{L}}{\partial t}=-1+\sum_{k=1}^{K} \mu_{k}=0 \\
\frac{\partial \mathcal{L}}{\partial \mathbf{w}_{i}}=\left(\sum_{k=1}^{K} \delta_{k} \cdot \mathbf{h}_{k}\right) s_{i}+\mu_{0} \cdot s_{i} s_{i}^{*} \mathbf{w}_{i}^{H}=\mathbf{0}, \forall i \in \mathcal{K} \\
\mathbf{h}_{k} \sum_{i=1}^{K} \mathbf{w}_{i} s_{i}-\lambda_{k} s_{k}=0, \forall k \in \mathcal{K} \\
\mu_{k}\left(t-\lambda_{k}\right)=0, \forall k \in \mathcal{K} \\
\mu_{0}\left(\sum_{i=1}^{K} s_{i}^{*} \mathbf{w}_{i}^{H} \mathbf{w}_{i} s_{i}-\frac{p_{0}}{K}\right)=0
\end{array}
$$

Based on (10b), it is firstly obtained that $\mu_{0} \neq 0$, and with the fact that $\mu_{0} \geq 0$ we can further obtain $\mu_{0}>0$. Then, $\mathbf{w}_{i}^{H}$ in (10b) can be expressed as

$$
\mathbf{w}_{i}^{H}=-\left(\sum_{k=1}^{K} \frac{\delta_{k}}{\mu_{0}} \cdot \mathbf{h}_{k}\right) \cdot \frac{1}{s_{i}^{*}}, \forall i \in \mathcal{K} .
$$

By introducing

$$
v_{k}=-\frac{\delta_{k}^{H}}{\mu_{0}}, \forall k \in \mathcal{K},
$$

the expression of $\mathbf{w}_{i}$ is obtained as

$$
\mathbf{w}_{i}=\left(\sum_{k=1}^{K} v_{k} \cdot \mathbf{h}_{k}^{H}\right) \cdot \frac{1}{s_{i}}, \forall k \in \mathcal{K} .
$$

Based on (13), we further obtain that

$$
\mathbf{w}_{i} s_{i}=\left(\sum_{k=1}^{K} v_{k} \cdot \mathbf{h}_{k}^{H}\right), \forall i \in \mathcal{K},
$$

which is a constant for any $i$. This mathematically verifies that the precoding vector for one symbol is a phase-rotated version of the precoding vector for another symbol. Then, with each $\mathbf{w}_{i}$ obtained, the precoding matrix $\mathbf{W}$ can be obtained and further expressed in a matrix form as

$$
\begin{aligned}
\mathbf{W} & =\left[\mathbf{w}_{1}, \mathbf{w}_{2}, \cdots, \mathbf{w}_{K}\right] \\
& =\left(\sum_{k=1}^{K} v_{k} \cdot \mathbf{h}_{k}^{H}\right) \cdot\left[\frac{1}{s_{1}}, \frac{1}{s_{2}}, \cdots, \frac{1}{s_{K}}\right] \\
& =\left[\mathbf{h}_{1}^{H}, \mathbf{h}_{2}^{H}, \cdots, \mathbf{h}_{K}^{H}\right]\left[v_{1}, v_{2}, \cdots, v_{K}\right]^{T}\left[\frac{1}{s_{1}}, \frac{1}{s_{2}}, \cdots, \frac{1}{s_{K}}\right] \\
& =\mathbf{H}^{H} \mathbf{\Upsilon} \hat{\mathbf{s}} .
\end{aligned}
$$

where we have introduced a column vector $\mathbf{\Upsilon} \overline{\overline{1}}$ $\left[v_{1}, v_{2}, \cdots, v_{K}\right]^{T}$ and a row vector $\hat{\mathbf{s}}=\left[\frac{1}{s_{1}}, \frac{1}{s_{2}}, \cdots, \frac{1}{s_{K}}\right]$. Subsequently, we further express (3) in a compact form as

$$
\mathbf{H W} \mathbf{s}=\operatorname{diag}(\boldsymbol{\Lambda}) \mathbf{s},
$$

where $\mathbf{H}=\left[\mathbf{h}_{1}^{T}, \mathbf{h}_{2}^{T}, \cdots, \mathbf{h}_{K}^{T}\right]^{T}$ is the channel matrix and $\boldsymbol{\Lambda}=\left[\lambda_{1}, \lambda_{2}, \cdots, \lambda_{K}\right]^{T}$. By substituting (15) into (16) and noting that $\hat{\mathbf{s}} \mathbf{s}=K$, we can further obtain

$$
\begin{aligned}
& \mathbf{H H}^{H} \mathbf{\Upsilon} \hat{\mathbf{s}} \mathbf{s}=\operatorname{diag}(\boldsymbol{\Lambda}) \mathbf{s} \\
\Rightarrow & \boldsymbol{\Upsilon}=\frac{1}{K} \cdot\left(\mathbf{H H}^{H}\right)^{-1} \operatorname{diag}(\boldsymbol{\Lambda}) \mathbf{s} .
\end{aligned}
$$

With (17), we can obtain the structure of the optimal precoding matrix as a function of scaling vector $\boldsymbol{\Lambda}$ as

$$
\mathbf{W}=\frac{1}{K} \cdot \mathbf{H}^{H}\left(\mathbf{H H}^{H}\right)^{-1} \operatorname{diag}(\mathbf{\Lambda}) \mathbf{s} \hat{\mathbf{s}} .
$$

It is easy to observe from (18) that the CI precoding is a symbol-level precoding scheme since the precoding matrix includes the expression of the symbol vector $\mathbf{s}$. Moreover, with (18) the original optimization problem on $\mathbf{W}$ is transformed into an optimization on the real-valued scaling vector $\boldsymbol{\Lambda}$. With the fact that $\mu_{0}>0$, based on (10e) we can obtain that the power constraint is strictly active, which leads to

$$
\begin{aligned}
& \|\mathbf{W} \mathbf{s}\|_{2}^{2}=p_{0} \\
\Rightarrow & \operatorname{tr}\left\{\mathbf{W} \mathbf{s s}{ }^{H} \mathbf{W}^{H}\right\}=p_{0} \\
\Rightarrow & \mathbf{s}^{H} \mathbf{W}^{H} \mathbf{W} \mathbf{s}=p_{0} \\
\Rightarrow & \frac{1}{K^{2}} \cdot \mathbf{s}^{H} \hat{\mathbf{s}}^{H} \mathbf{s}^{H} \operatorname{diag}(\mathbf{\Lambda})\left(\mathbf{H} \mathbf{H}^{H}\right)^{-1} \operatorname{diag}(\mathbf{\Lambda}) \mathbf{s} \hat{\mathbf{s}}=p_{0} \\
\Rightarrow & \mathbf{s}^{H} \operatorname{diag}(\mathbf{\Lambda})\left(\mathbf{H H}^{H}\right)^{-1} \operatorname{diag}(\boldsymbol{\Lambda}) \mathbf{s}=p_{0} \\
\Rightarrow & \boldsymbol{\Lambda}^{T} \operatorname{diag}\left(\mathbf{s}^{H}\right)\left(\mathbf{H H}^{H}\right)^{-1} \operatorname{diag}(\mathbf{s}) \boldsymbol{\Lambda}=p_{0} \\
\Rightarrow & \boldsymbol{\Lambda}^{T} \mathbf{T} \boldsymbol{\Lambda}=p_{0}
\end{aligned}
$$

where we note that $\lambda_{k}^{H}=\lambda_{k}$ as each $\lambda_{k}$ is real, and $\mathbf{T}$ is defined as

$$
\mathbf{T}=\operatorname{diag}\left(\mathbf{s}^{H}\right)\left(\mathbf{H H}^{H}\right)^{-1} \operatorname{diag}(\mathbf{s}) .
$$

It is easy to obtain that $\mathbf{T}$ is Hermitian and positive semidefinite, which further leads to

$$
\boldsymbol{\Lambda}^{T} \mathbf{T} \boldsymbol{\Lambda}=\boldsymbol{\Lambda}^{T} \Re(\mathbf{T}) \boldsymbol{\Lambda}=\boldsymbol{\Lambda}^{T} \mathbf{V} \boldsymbol{\Lambda}=p_{0},
$$

where $\mathbf{V}=\Re(\mathbf{T})$ is a symmetric and positive semi-definite matrix. With (21) obtained, we can formulate a new convex optimization problem on $\boldsymbol{\Lambda}$ that is equivalent to the original optimization $\mathcal{P}_{1}$, expressed as

$$
\begin{array}{ll}
\mathcal{P}_{3}: & \min _{\boldsymbol{\Lambda}, t}-t \\
\text { s.t. } & \boldsymbol{\Lambda}^{T} \mathbf{V} \boldsymbol{\Lambda}-p_{0}=0 \\
& t-\lambda_{k} \leq 0, \forall k \in \mathcal{K}
\end{array}
$$

The optimal precoding matrix for the original problem $\mathcal{P}_{1}$ in (5) can be obtained with (18) based on the obtained $\boldsymbol{\Lambda}$ by solving $\mathcal{P}_{3}$. In the following, we analyze the convex optimization $\mathcal{P}_{3}$ with the Lagrangian approach, where the Lagrangian of $\mathcal{P}_{3}$ is formulated as

$$
\begin{aligned}
& \mathcal{L}\left(\boldsymbol{\Lambda}, t, \alpha_{0}, \mu_{k}\right)=-t+\alpha_{0}\left(\boldsymbol{\Lambda}^{T} \mathbf{V} \boldsymbol{\Lambda}-p_{0}\right)+\sum_{k=1}^{K} \mu_{k}\left(t-\lambda_{k}\right) \\
& =\left(\mathbf{1}^{T} \mathbf{u}-1\right) t+\alpha_{0} \cdot \boldsymbol{\Lambda}^{T} \mathbf{V} \boldsymbol{\Lambda}-\mathbf{u}^{T} \boldsymbol{\Lambda}-\alpha_{0} p_{0},
\end{aligned}
$$

where $\alpha_{0}$ and $\mu_{k}$ are the dual variables and $\mu_{k} \geq 0, \forall k \in \mathcal{K}$. $\mathbf{u}=\left[\mu_{1}, \mu_{2}, \cdots, \mu_{K}\right]^{T}$ is a column vector that consists of the dual variables and the vector $\mathbf{1}=[1,1, \cdots, 1]^{T}$. Based on 
(23), the KKT conditions of $\mathcal{P}_{3}$ for optimality are expressed as

$$
\begin{array}{r}
\frac{\partial \mathcal{L}}{\partial t}=\mathbf{1}^{T} \mathbf{u}-1=0 \\
\frac{\partial \mathcal{L}}{\partial \boldsymbol{\Lambda}}=\alpha_{0}\left(\mathbf{V}+\mathbf{V}^{T}\right) \boldsymbol{\Lambda}-\mathbf{u}=\mathbf{0} \\
\boldsymbol{\Lambda}^{T} \mathbf{V} \boldsymbol{\Lambda}-p_{0}=0 \\
\mu_{k}\left(t-\lambda_{k}\right)=0, \forall k \in \mathcal{K}
\end{array}
$$

Based on (24b), firstly we have $\alpha_{0} \neq 0$, and we can further obtain the expression of $\Lambda$, given by

$$
\boldsymbol{\Lambda}=\frac{1}{2 \alpha_{0}} \mathbf{V}^{-1} \mathbf{u},
$$

where we note that $\mathbf{V}$ is symmetric. By substituting the expression of $\boldsymbol{\Lambda}$ in (25) into (24c), we can express $\alpha_{0}$ as a function of the dual vector $\mathbf{u}$, given by

$$
\begin{aligned}
& \left(\frac{1}{2 \alpha_{0}} \mathbf{V}^{-1} \mathbf{u}\right)^{T} \mathbf{V}\left(\frac{1}{2 \alpha_{0}} \mathbf{V}^{-1} \mathbf{u}\right)=p_{0} \\
\Rightarrow & \frac{1}{4 \alpha_{0}^{2}} \mathbf{u}^{T} \mathbf{V}^{-1} \mathbf{V} \mathbf{V}^{-1} \mathbf{u}=p_{0} \\
\Rightarrow & \alpha_{0}=\sqrt{\frac{\mathbf{u}^{T} \mathbf{V}^{-1} \mathbf{u}}{4 p_{0}}} .
\end{aligned}
$$

For the convex optimization $\mathcal{P}_{3}$ in (22), it is easy to verify that the Slater's condition is satisfied [39], which means that the dual gap is zero. Therefore, we can solve $\mathcal{P}_{3}$ by solving its corresponding dual problem, which is given by

$$
\mathcal{U}=\max _{\mathbf{u}, \alpha_{0}} \min _{\boldsymbol{\Lambda}, t} \mathcal{L}\left(\boldsymbol{\Lambda}, t, \alpha_{0}, \mathbf{u}\right) .
$$

For the dual problem $\mathcal{U}$, the inner minimization is achieved with (24a) and the obtained $\boldsymbol{\Lambda}$ in (25), and therefore the dual problem can be further transformed into

$$
\begin{aligned}
\mathcal{U}= & \max _{\mathbf{u}, \alpha_{0}} \alpha_{0}\left(\frac{1}{2 \alpha_{0}} \mathbf{V}^{-1} \mathbf{u}\right)^{T} \mathbf{V}\left(\frac{1}{2 \alpha_{0}} \mathbf{V}^{-1} \mathbf{u}\right) \\
& -\mathbf{u}^{T}\left(\frac{1}{2 \alpha_{0}} \mathbf{V}^{-1} \mathbf{u}\right)-\alpha_{0} p_{0} \\
= & \max _{\mathbf{u}, \alpha_{0}} \frac{1}{4 \alpha_{0}} \mathbf{u}^{T} \mathbf{V}^{-1} \mathbf{V} \mathbf{V}^{-1} \mathbf{u}-\frac{1}{2 \alpha_{0}} \mathbf{u}^{T} \mathbf{V}^{-1} \mathbf{u}-\alpha_{0} p_{0} \\
= & \max _{\mathbf{u}, \alpha_{0}}-\frac{1}{4 \alpha_{0}} \mathbf{u}^{T} \mathbf{V}^{-1} \mathbf{u}-\alpha_{0} p_{0} \\
= & \max _{\mathbf{u}}-\frac{\mathbf{u}^{T} \mathbf{V}^{-1} \mathbf{u}}{4 \sqrt{\frac{\mathbf{u}^{T} \mathbf{V}^{-1} \mathbf{u}}{4 p_{0}}}}-\sqrt{\frac{\mathbf{u}^{T} \mathbf{V}^{-1} \mathbf{u}}{4 p_{0}}} \cdot p_{0} \\
= & \max _{\mathbf{u}}-\sqrt{p_{0} \cdot \mathbf{u}^{T} \mathbf{V}^{-1} \mathbf{u}} .
\end{aligned}
$$

Due to the fact that $y=\sqrt{x}$ is a monotonic function, therefore the dual problem $\mathcal{U}$ is equivalent to the following optimization problem

$$
\begin{array}{ll}
\mathcal{P}_{4}: & \min _{\mathbf{u}} \mathbf{u}^{T} \mathbf{V}^{-1} \mathbf{u} \\
\text { s.t. } & \mathbf{1}^{T} \mathbf{u}=1 \\
& \mu_{k} \geq 0, \forall k \in \mathcal{K}
\end{array}
$$

where the first constraint comes from (24a).
Based on our analysis and transformations above, we have transformed and simplified the original problem, and shown that the original optimization can be solved by solving $\mathcal{P}_{4}$. To be more specific, through (26), (25) and (18), we arrive at a final closed-form expression of the optimal precoding matrix as a function of $\mathbf{u}$, given by

$$
\mathbf{W}=\frac{1}{K} \mathbf{H}^{H}\left(\mathbf{H H}^{H}\right)^{-1} \operatorname{diag}\left\{\sqrt{\frac{p_{0}}{\mathbf{u}^{T} \mathbf{V}^{-1} \mathbf{u}}} \mathbf{V}^{-1} \mathbf{u}\right\} \mathbf{s} \hat{\mathbf{s}} .
$$

Moreover, it is observed that $\mathcal{P}_{4}$ is a typical QP optimization problem over a simplex, and it has already been shown in the existing literature that this optimization can be more efficiently solved than SOCP with a similar problem size using the simplex or interior-point methods [40]-[42]. This validates that the complexity of CI-based precoding will be reduced with our derivations by transforming the original SOCP optimization into a QP optimization with an equal or smaller variable size, where we note that the size of the variable $\mathbf{w}_{i}$ in the original optimization is $N_{t} \times 1$, while the variable size in our QP formulation is $\mathbf{u} \in \mathcal{R}^{K \times 1}$, with $K \leq N_{t}$.

\section{B. Non-Strict Phase Rotation}

We extend our analysis to the case of non-strict phase rotation. Similarly, before formulating the optimization problem, we firstly construct the condition that the precoding designs should satisfy such that the received signals are located in the constructive region. Based on Fig. 3, for consistency we denote $\overrightarrow{O A}=t \cdot s_{k}$ and $t=|\overrightarrow{O A}|$ is the objective to be maximized. Following (2), we denote the received signal for user $k$ as $\overrightarrow{O B}$, which is expressed as

$$
\overrightarrow{O B}=\mathbf{h}_{k} \mathbf{W} \mathbf{s}=\lambda_{k} s_{k} .
$$

In the case of non-strict phase rotation, each $\lambda_{k}$ can be a complex value, which mathematically represents that a phase rotation is included for the received signal $\overrightarrow{O B}$ compared to the data symbol $s_{k}$, as shown in Fig. 3. This is different from the case of strict phase rotation where each $\lambda_{k}$ is strictly real. Then, based on the fact that $\overrightarrow{O C}$ and $\overrightarrow{C B}$ are perpendicular, we can obtain the expression of $\overrightarrow{O C}$ and $\overrightarrow{C B}$, given by

$$
\overrightarrow{O C}=\Re\left(\lambda_{k}\right) s_{k}=\lambda_{k}^{\Re} s_{k}, \overrightarrow{C B}=j \cdot \Im\left(\lambda_{k}\right) s_{k}=j \cdot \lambda_{k}^{\Im} s_{k},
$$

where based on Fig. 3 the imaginary unit ' $j$ ' denotes a phase rotation of $90^{\circ}$ geometrically. For simplicity of denotation, we denote $\lambda_{k}^{\Re}=\Re\left(\lambda_{k}\right)$ and $\lambda_{k}^{\Im}=\Im\left(\lambda_{k}\right)$, respectively. Due to the fact that the nodes ' $\mathrm{O}$ ', 'A' and ' $\mathrm{C}$ ' are co-linear, we can further obtain the expression of $\overrightarrow{A C}$ as

$$
\overrightarrow{A C}=\left(\lambda_{k}^{\Re}-t\right) s_{k} .
$$

In Fig. 3, we can observe that to have the received signal $\overrightarrow{O B}$ located in the constructive region is equivalent to the following condition:

$$
\begin{aligned}
& \theta_{A B} \leq \theta_{t} \\
\Rightarrow & \tan \theta_{A B} \leq \tan \theta_{t} \\
\Rightarrow & \frac{|\overrightarrow{C B}|}{|\overrightarrow{A C}|}=\frac{\left|\lambda_{k}^{\Im} s_{k}\right|}{\left|\left(\lambda_{k}^{\Re}-t\right) s_{k}\right|} \leq \tan \theta_{t} \\
\Rightarrow & \left(\lambda_{k}^{\Re}-t\right) \tan \theta_{t} \geq\left|\lambda_{k}^{\Im}\right| .
\end{aligned}
$$


In the case of $\lambda_{k}^{\Im}=0, \forall k \in \mathcal{K}$, (34) is identical to (4), and the non-strict phase rotation reduces to the strict phase rotation. For $\mathcal{M}$-PSK modulation, it is observed from the modulation constellation that the threshold angle $\theta_{t}$ can be expressed as

$$
\theta_{t}=\frac{\pi}{\mathcal{M}} \text {. }
$$

With the above formulation, we can construct the optimization problem of CI for non-strict phase rotation as

$$
\begin{array}{ll}
\mathcal{P}_{5}: & \max _{\mathbf{W}, t} t \\
\text { s.t. } & \mathbf{h}_{k} \mathbf{W} \mathbf{s}=\lambda_{k} s_{k}, \forall k \in \mathcal{K} \\
& \left(\lambda_{k}^{\Re}-t\right) \tan \theta_{t} \geq\left|\lambda_{k}^{\Im}\right|, \forall k \in \mathcal{K} \\
& \|\mathbf{W} \mathbf{s}\|_{2}^{2} \leq p_{0}
\end{array}
$$

To further analyze the optimization problem for non-strict phase rotation, we first transform $\mathcal{P}_{5}$ in (36) into a standard minimization form, given by

$$
\begin{array}{ll}
\mathcal{P}_{6}: & \min _{\mathbf{W}, t}-t \\
\text { s.t. } & \mathbf{h}_{k} \mathbf{W} \mathbf{s}-\lambda_{k} s_{k}=0, \forall k \in \mathcal{K} \\
& \left|\lambda_{k}^{\Im}\right|-\left(\lambda_{k}^{\Re}-t\right) \tan \theta_{t} \leq 0, \forall k \in \mathcal{K} \\
& \sum_{i=1}^{K} s_{i}^{*} \mathbf{w}_{i}^{H} \mathbf{w}_{i} s_{i}-\frac{p_{0}}{K} \leq 0
\end{array}
$$

Then, by following a similar step in (9)-(17) with the Lagrangian approach, we can obtain that the optimal precoding structure for non-strict phase rotation is the same as that for strict phase rotation, which is given in (18). With the power constraint strictly active, we can further obtain that

$$
\begin{aligned}
& \|\mathbf{W} \mathbf{s}\|_{2}^{2}=p_{0} \\
\Rightarrow & \mathbf{s}^{H} \mathbf{W}^{H} \mathbf{W} \mathbf{s}=p_{0} \\
\Rightarrow & \mathbf{s}^{H} \operatorname{diag}\left(\mathbf{\Lambda}^{H}\right)\left(\mathbf{H H}^{H}\right)^{-1} \operatorname{diag}(\boldsymbol{\Lambda}) \mathbf{s}=p_{0} \\
\Rightarrow & \boldsymbol{\Lambda}^{H} \operatorname{diag}\left(\mathbf{s}^{H}\right)\left(\mathbf{H} \mathbf{H}^{H}\right)^{-1} \operatorname{diag}(\mathbf{s}) \boldsymbol{\Lambda}=p_{0} \\
\Rightarrow & \boldsymbol{\Lambda}^{H} \mathbf{T} \boldsymbol{\Lambda}=p_{0},
\end{aligned}
$$

where $\mathbf{T}$ is given by (20). However, we note that, different from the case of strict phase rotation, for the case of nonstrict phase rotation (38) is not in a quadratic form since each $\lambda_{k}$ can be complex. By decomposing

$$
\hat{\boldsymbol{\Lambda}}=\left[\Re\left(\boldsymbol{\Lambda}^{T}\right), \Im\left(\boldsymbol{\Lambda}^{T}\right)\right]^{T}, \hat{\mathbf{T}}=\left[\begin{array}{cc}
\Re(\mathbf{T}) & -\Im(\mathbf{T}) \\
\Im(\mathbf{T}) & \Re(\mathbf{T})
\end{array}\right],
$$

we can expand (38) with its real and imaginary components and further transform the power constraint into a quadratic form, given by

$$
\begin{aligned}
& \|\mathbf{W} \mathbf{s}\|_{2}^{2}=p_{0} \\
\Rightarrow & \hat{\boldsymbol{\Lambda}}^{T} \hat{\mathbf{T}} \hat{\boldsymbol{\Lambda}}-p_{0}=0 .
\end{aligned}
$$

Similar to the optimization $\mathcal{P}_{3}$ in (22) for strict phase rotation, we can formulate an optimization problem on $\hat{\Lambda}$ for non-strict phase rotation, expressed as

$$
\begin{array}{ll}
\mathcal{P}_{7}: & \min _{\hat{\boldsymbol{\Lambda}}, t}-t \\
\text { s.t. } & \hat{\boldsymbol{\Lambda}}^{T} \hat{\mathbf{T}} \hat{\boldsymbol{\Lambda}}-p_{0}=0 \\
& \frac{\lambda_{k}^{\Im}}{\tan \theta_{t}}+t-\lambda_{k}^{\Re} \leq 0, \forall k \in \mathcal{K} \\
& -\frac{\lambda_{k}^{\Im}}{\tan \theta_{t}}+t-\lambda_{k}^{\Re} \leq 0, \forall k \in \mathcal{K}
\end{array}
$$

where we have transformed the CI constraint with the absolute value on $\lambda_{k}^{\Im}$ into two separate constraints. We then analyze $\mathcal{P}_{7}$ with Lagrangian and KKT conditions, where the Lagrangian of $\mathcal{P}_{7}$ is constructed as

$$
\begin{aligned}
\mathcal{L} & \left(\hat{\boldsymbol{\Lambda}}, t, \hat{\alpha}_{0}, \hat{\mu}_{k}, \hat{\nu}_{k}\right)=-t+\hat{\alpha}_{0}\left(\hat{\boldsymbol{\Lambda}}^{T} \hat{\mathbf{T}} \hat{\boldsymbol{\Lambda}}-p_{0}\right) \\
& +\sum_{k=1}^{K} \hat{\mu}_{k}\left(\frac{\lambda_{k}^{\Im}}{\tan \theta_{t}}+t-\lambda_{k}^{\Re}\right)+\sum_{k=1}^{K} \hat{\nu}_{k}\left(-\frac{\lambda_{k}^{\Im}}{\tan \theta_{t}}+t-\lambda_{k}^{\Re}\right) \\
= & {\left[\sum_{k=1}^{K}\left(\hat{\mu}_{k}+\hat{\nu}_{k}\right)-1\right] t+\hat{\alpha}_{0} \hat{\boldsymbol{\Lambda}}^{T} \hat{\mathbf{T}} \hat{\boldsymbol{\Lambda}}-\hat{\alpha}_{0} p_{0} } \\
& -\sum_{k=1}^{K}\left(\hat{\mu}_{k}+\hat{\nu}_{k}\right) \lambda_{k}^{\Re}+\sum_{k=1}^{K}\left(\hat{\mu}_{k}-\hat{\nu}_{k}\right) \frac{\lambda_{k}^{\Im}}{\tan \theta_{t}},
\end{aligned}
$$

where $\hat{\alpha}_{0}, \hat{\mu}_{k}$ and $\hat{\nu}_{k}$ are the dual variables, and $\hat{\mu}_{k} \geq 0$, $\hat{\nu}_{k} \geq 0, \forall k$. By introducing

$$
\begin{aligned}
& \hat{\mathbf{u}}=\left[\hat{\mu}_{1}, \hat{\mu}_{2}, \cdots, \hat{\mu}_{K}, \hat{\nu}_{1}, \hat{\nu}_{2}, \cdots, \hat{\nu}_{K}\right]^{T}, \\
& \mathbf{S}=\left[\begin{array}{cc}
\mathbf{I} & -\frac{1}{\tan \theta_{t}} \cdot \mathbf{I} \\
\mathbf{I} & \frac{1}{\tan \theta_{t}} \cdot \mathbf{I}
\end{array}\right],
\end{aligned}
$$

where $\hat{\mathbf{u}} \in \mathcal{C}^{2 K \times 1}$ and $\mathbf{S} \in \mathcal{C}^{2 K \times 2 K}$, the Lagrangian for $\mathcal{P}_{7}$ can be further simplified into

$\mathcal{L}\left(\hat{\mathbf{\Lambda}}, t, \hat{\alpha}_{0}, \hat{\mathbf{u}}\right)=\left(\mathbf{1}^{T} \hat{\mathbf{u}}-1\right) t+\hat{\alpha}_{0} \hat{\boldsymbol{\Lambda}}^{T} \hat{\mathbf{T}} \hat{\mathbf{\Lambda}}-\hat{\mathbf{u}}^{T} \mathbf{S} \hat{\mathbf{\Lambda}}-\hat{\alpha}_{0} p_{0}$.

Based on (44), we express the KKT conditions for optimality of $\mathcal{P}_{7}$ in the following:

$$
\begin{array}{r}
\frac{\partial \mathcal{L}}{\partial t}=\mathbf{1}^{T} \hat{\mathbf{u}}-1=0 \\
\frac{\partial \mathcal{L}}{\partial \hat{\mathbf{\Lambda}}}=2 \hat{\alpha}_{0} \hat{\mathbf{T}} \hat{\boldsymbol{\Lambda}}-\mathbf{S}^{T} \hat{\mathbf{u}}=\mathbf{0} \\
\hat{\boldsymbol{\Lambda}}^{T} \hat{\mathbf{T}} \hat{\mathbf{\Lambda}}-p_{0}=0 \\
\hat{\mu}_{k}\left(\frac{\lambda_{k}^{\Im}}{\tan \theta_{t}}+t-\lambda_{k}^{\Re}\right)=0, \forall k \in \mathcal{K} \\
\hat{\nu}_{k}\left(-\frac{\lambda_{k}^{\Im}}{\tan \theta_{t}}+t-\lambda_{k}^{\Re}\right)=0, \forall k \in \mathcal{K}
\end{array}
$$

Based on (45b) we can obtain $\hat{\alpha}_{0} \neq 0$ and the expression of $\hat{\Lambda}$, given by

$$
\hat{\mathbf{\Lambda}}=\frac{1}{2 \hat{\alpha}_{0}} \hat{\mathbf{T}}^{-1} \mathbf{S}^{T} \hat{\mathbf{u}}
$$

where we note that $\hat{\mathbf{T}}$ is symmetric. Moreover, similar to the case of strict phase rotation, by substituting the expression of 
$\hat{\Lambda}$ in (46) into (45c), we can further obtain the expression of $\hat{\alpha}_{0}$, given by

$$
\begin{aligned}
& \left(\frac{1}{2 \hat{\alpha}_{0}} \hat{\mathbf{T}} \mathbf{S}^{T} \hat{\mathbf{u}}\right)^{T} \hat{\mathbf{T}}\left(\frac{1}{2 \hat{\alpha}_{0}} \hat{\mathbf{T}} \mathbf{S}^{T} \hat{\mathbf{u}}\right)=p_{0} \\
\Rightarrow & \hat{\alpha}_{0}=\sqrt{\frac{\hat{\mathbf{u}}^{T} \mathbf{S} \hat{\mathbf{T}}^{-1} \mathbf{S}^{T} \hat{\mathbf{u}}}{4 p_{0}}}=\sqrt{\frac{\hat{\mathbf{u}}^{T} \hat{\mathbf{V}}^{-1} \hat{\mathbf{u}}}{4 p_{0}}},
\end{aligned}
$$

where for simplicity and consistency we introduce

$$
\hat{\mathbf{V}}^{-1}=\mathbf{S} \hat{\mathbf{T}}^{-1} \mathbf{S}^{T} \text {. }
$$

Similar to the case for strict phase rotation, it is easy to observe that the Slater's condition is satisfied for $\mathcal{P}_{7}$, and therefore by following a similar approach in (27) and (28), the dual problem for $\mathcal{P}_{7}$ can be formulated into

$$
\hat{\mathcal{U}}=\max _{\hat{\mathbf{u}}}-\sqrt{p_{0} \cdot \hat{\mathbf{u}}^{T} \hat{\mathbf{V}}^{-1} \hat{\mathbf{u}}},
$$

which further leads to the following equivalent optimization for non-strict phase rotation

$$
\begin{array}{ll}
\mathcal{P}_{8}: & \min _{\hat{\mathbf{u}}} \hat{\mathbf{u}}^{T} \hat{\mathbf{V}}^{-1} \hat{\mathbf{u}} \\
\text { s.t. } & \mathbf{1}^{T} \hat{\mathbf{u}}=1 \\
& \hat{u}_{k} \geq 0, \forall k \in\{1,2, \cdots, 2 K\}
\end{array}
$$

where we denote $\hat{u}_{k}$ as the $k$-th entry in $\hat{\mathbf{u}}$, and we obtain $\hat{\mathbf{V}}^{-1} \in \mathcal{C}^{2 K \times 2 K}$ based on (48). $\mathcal{P}_{8}$ is also a QP optimization over a simplex, which can be efficiently solved. The final optimal precoding matrix for non-strict phase rotation can be similarly obtained in a closed form as a function of $\hat{\mathbf{u}}$, given by

$$
\mathbf{W}=\frac{1}{K} \mathbf{H}^{H}\left(\mathbf{H} \mathbf{H}^{H}\right)^{-1} \operatorname{diag}\left\{\sqrt{\frac{p_{0}}{\hat{\mathbf{u}}^{T} \hat{\mathbf{V}}^{-1} \hat{\mathbf{u}}}} \mathbf{U} \hat{\mathbf{T}}^{-1} \mathbf{S}^{T} \hat{\mathbf{u}}\right\} \mathbf{s} \hat{\mathbf{s}},
$$

where $\mathbf{U}=\left[\begin{array}{ll}\mathbf{I} & j \cdot \mathbf{I}\end{array}\right]$ is a transformation matrix that transform the real-valued vector $\hat{\Lambda}$ into its complex equivalence.

Based on the formulated equivalent optimization problems $\mathcal{P}_{4}$ in (29) and $\mathcal{P}_{8}$ in (50), we note the similarity of the optimization problem for strict phase rotation and non-strict phase rotation. We observe that the objective function of $\mathcal{P}_{4}$ for strict phase rotation and $\mathcal{P}_{8}$ for non-strict phase rotation is identical, and both optimization problems share the same constraints. It is further observed that the only difference between $\mathcal{P}_{4}$ and $\mathcal{P}_{8}$ is the problem size. It is then concluded that a $K$-dimensional optimization problem for non-strict phase rotation and a $2 K$ dimensional optimization for strict phase rotation share the same problem formulation, and therefore they can be solved in a similar way.

\section{CI AS a Generalization of ZF PRECODING}

In this section, we discuss the connection between the CI precoding for strict phase rotation and the conventional ZF precoding. For the $\mathrm{CI}$ precoding with non-strict phase rotation, the connection can be obtained in a similar way. To compare the CI precoding and the conventional ZF precoding, as a reference we first present the precoded signal vector of $\mathrm{ZF}$, given by

$$
\mathbf{x}_{Z F}=\mathbf{W}_{Z F} \mathbf{s}=\frac{1}{f} \cdot \mathbf{H}^{H}\left(\mathbf{H} \mathbf{H}^{H}\right)^{-1} \mathbf{s}
$$

where $f$ is the scaling factor to meet the transmit power constraint. For fairness of comparison, we employ a symbollevel normalization for $\mathbf{W}_{Z F}$ such that $\left\|\mathbf{W}_{Z F} \mathbf{s}\right\|_{2}^{2}=p_{0}$ as for the considered CI precoding, which leads to the expression of $f$ as

$$
f=\sqrt{\frac{\left\|\mathbf{W}_{Z F} \mathbf{s}\right\|_{2}^{2}}{p_{0}}}=\sqrt{\frac{\mathbf{s}^{H}\left(\mathbf{H H}^{H}\right)^{-1} \mathbf{s}}{p_{0}}} .
$$

By denoting $\mathbf{C}=\left(\mathbf{H} \mathbf{H}^{H}\right)^{-1}$, the expression of $f$ can be further transformed into

$$
\begin{array}{r}
f=\sqrt{\frac{\sum_{m=1}^{K} \sum_{n=1}^{K} \mathbf{C}(m, n) s_{m}^{*} s_{n}}{p_{0}}} \\
\Rightarrow \sum_{m=1}^{K} \sum_{n=1}^{K} \mathbf{C}(m, n) s_{m}^{*} s_{n}=f^{2} p_{0} .
\end{array}
$$

Subsequently, we perform the mathematical analysis on the optimization problem $\mathcal{P}_{4}$ on $\mathbf{u}$ for strict phase rotation. By applying the Lagrangian approach, we can obtain the Lagrangian of $\mathcal{P}_{4}$, given by

$$
\begin{aligned}
\mathcal{L}\left(\mathbf{u}, q_{0}, \mathbf{q}\right) & =\mathbf{u}^{T} \mathbf{V}^{-1} \mathbf{u}+q_{0}\left(\mathbf{1}^{T} \mathbf{u}-1\right)-\sum_{k=1}^{K} q_{k} \mu_{k} \\
& =\mathbf{u}^{T} \mathbf{V}^{-1} \mathbf{u}+q_{0} \cdot \mathbf{1}^{T} \mathbf{u}-\mathbf{q}^{T} \mathbf{u}-q_{0}
\end{aligned}
$$

where the vector $\mathbf{q}=\left[q_{1}, q_{2}, \cdots, q_{K}\right]^{T}$ consists of each nonnegative dual variable $q_{k}$ of $\mathcal{P}_{4}$. Based on (55), we express the KKT conditions of $\mathcal{P}_{4}$ as

$$
\begin{array}{r}
\frac{\partial \mathcal{L}}{\partial \mathbf{u}}=2 \mathbf{V}^{-1} \mathbf{u}+q_{0} \cdot \mathbf{1}-\mathbf{q}=\mathbf{0} \\
\mathbf{1}^{T} \mathbf{u}-1=0 \\
q_{k} \mu_{k}=0, \forall k \in \mathcal{K}
\end{array}
$$

Based on (56a) we can obtain the expression of $\mathbf{u}$ as a function of the dual variables, given by

$$
\mathbf{u}=\frac{1}{2} \mathbf{V}\left(\mathbf{q}-q_{0} \cdot \mathbf{1}\right)
$$

and each $\mu_{k}$ as

$$
\mu_{k}=\frac{1}{2}\left(\mathbf{v}_{k} \mathbf{q}-q_{0} a_{k}\right), \forall k \in \mathcal{K},
$$

where we have decomposed $\mathbf{V}$ into $\mathbf{V}=\left[\mathbf{v}_{1}^{T}, \mathbf{v}_{2}^{T}, \cdots, \mathbf{v}_{K}^{T}\right]^{T}$. $\mathbf{a}=\left[a_{1}, a_{2}, \cdots, a_{K}\right]^{T}$ denotes the column vector obtained from the sum of $\mathbf{V}$ by column, with each entry given by

$$
a_{k}=\sum_{i=1}^{K} \mathbf{V}(k, i) .
$$


By substituting the expression of $\mathbf{u}$ into (56b), we further obtain that

$$
\begin{aligned}
& \mathbf{1}^{T} \mathbf{u}-1=0 \\
\Rightarrow & \frac{1}{2} \sum_{k=1}^{K}\left\{\left[\sum_{i=1}^{K} \mathbf{V}(k, i) q_{i}\right]-q_{0} a_{k}\right\}-1=0 \\
\Rightarrow & \frac{1}{2} \sum_{i=1}^{K}\left[\sum_{k=1}^{K} \mathbf{V}(k, i)\right] q_{i}-\frac{1}{2} q_{0} c-1=0 \\
\Rightarrow & \frac{1}{2} \sum_{i=1}^{K} b_{i} q_{i}-\frac{1}{2} q_{0} c-1=0 \\
\Rightarrow & q_{0}=\frac{\mathbf{b q}-2}{c}
\end{aligned}
$$

where $\mathbf{b}$ is a row vector obtained from the sum of $\mathbf{V}$ by row and $c$ denotes the sum of all the entries in $\mathbf{V}$. $\mathbf{b}$ and $c$ are given by

$$
\begin{aligned}
& \mathbf{b}=\sum_{k=1}^{K} \mathbf{v}_{k}=\mathbf{a}^{T}, \\
& c=\sum_{k=1}^{K} \sum_{i=1}^{K} \mathbf{V}(k, i)=\mathbf{a}^{T} \mathbf{1},
\end{aligned}
$$

where $\mathbf{b}=\mathbf{a}^{T}$ is based on the fact that $\mathbf{V}$ is symmetric. By substituting the expression of $q_{0}$ in (60) into (58), the expression of each $\mu_{k}$ can be further transformed into

$$
\begin{aligned}
\mu_{k} & =\frac{1}{2} \mathbf{v}_{k} \mathbf{q}-\frac{a_{k}}{2} \frac{\mathbf{a}^{T} \mathbf{q}-2}{c} \\
& =\frac{1}{2}\left(\mathbf{v}_{k}-\frac{a_{k}}{c} \mathbf{a}^{T}\right) \mathbf{q}+\frac{a_{k}}{c},
\end{aligned}
$$

which further leads to the expression of $\mathbf{u}$ as

$$
\mathbf{u}=\frac{1}{2}(\mathbf{V}-\mathbf{\Phi}) \mathbf{q}+\frac{\mathbf{a}}{c}
$$

where $\boldsymbol{\Phi}=\frac{\mathbf{a a}^{T}}{c}$. By substituting the expression of $\mathbf{u}$ into the expression of $\boldsymbol{\Lambda}$ in (25), we can further obtain that

$$
\begin{aligned}
\boldsymbol{\Lambda} & =\frac{1}{2 \alpha_{0}} \mathbf{V}^{-1}\left[\frac{1}{2}(\mathbf{V}-\mathbf{\Phi}) \mathbf{q}+\frac{\mathbf{a}}{c}\right] \\
& =\frac{1}{2 \alpha_{0} c} \mathbf{V}^{-1} \mathbf{a}+\frac{1}{4 \alpha_{0}}\left(\mathbf{I}-\mathbf{V}^{-1} \mathbf{\Phi}\right) \mathbf{q} \\
& =\frac{1}{2 \alpha_{0} c} \mathbf{m}+\frac{1}{4 \alpha_{0}}\left(\mathbf{I}-\mathbf{V}^{-1} \mathbf{\Phi}\right) \mathbf{q},
\end{aligned}
$$

where we have defined

$$
\mathbf{m}=\mathbf{V}^{-1} \mathbf{a} .
$$

In (65), $\mathbf{m} \in \mathcal{C}^{K \times 1}$ and $\mathbf{m}=\left[m_{1}, m_{2}, \cdots, m_{K}\right]^{T}$. Based on the expression of $\mathbf{a}$, each $m_{k}$ is obtained as

$$
\begin{aligned}
m_{k} & =\sum_{n=1}^{K} \mathbf{V}^{-1}(k, n) a_{n} \\
& =\sum_{n=1}^{K} \mathbf{V}^{-1}(k, n) \sum_{i=1}^{K} \mathbf{V}(n, i) \\
& =\sum_{n=1}^{K} \mathbf{V}^{-1}(k, n) \mathbf{V}(n, k)+\sum_{i \neq k} \sum_{n=1}^{K} \mathbf{V}^{-1}(k, n) \mathbf{V}(n, i) \\
& =1+\sum_{i \neq k} 0 \\
& =1,
\end{aligned}
$$

which also means that $\mathbf{m}=\mathbf{V}^{-1} \mathbf{a}=\mathbf{1}$. With this fact, the expression of $\boldsymbol{\Lambda}$ is further transformed into

$$
\boldsymbol{\Lambda}=\frac{1}{2 \alpha_{0} c} \mathbf{1}+\frac{1}{4 \alpha_{0}}\left(\mathbf{I}-\mathbf{V}^{-1} \boldsymbol{\Phi}\right) \mathbf{q}
$$

based on which we shall discuss the connection between the CI precoding and the conventional ZF scheme. In (67), if we set

$$
q_{k}=0, \forall k \in \mathcal{K}
$$

based on (63) we can obtain that

$$
\mathbf{u}=\frac{\mathbf{a}}{c}
$$

and based on (26) we further obtain that

$$
\alpha_{0}=\sqrt{\frac{\mathbf{a}^{T} \mathbf{V}^{-1} \mathbf{a}}{4 c^{2} p_{0}}}=\sqrt{\frac{\mathbf{a}^{T} \mathbf{1}}{4 c^{2} p_{0}}}=\sqrt{\frac{c}{4 c^{2} p_{0}}}=\frac{1}{2 \sqrt{c p_{0}}} .
$$

Then, the expression of $\boldsymbol{\Lambda}$ is simplified into

$$
\boldsymbol{\Lambda}=\frac{1}{2 \alpha_{0} c} \mathbf{1}=\frac{2 \sqrt{c p_{0}}}{2 c}=\sqrt{\frac{p_{0}}{c}} .
$$

Based on the expression of $\mathbf{T}$ in (20), we can obtain the expression of $\mathbf{T}(m, n)$ as

$$
\mathbf{T}(m, n)=\mathbf{C}(m, n) s_{m}^{*} s_{n},
$$

and with the fact that $\mathbf{T}$ is Hermitian, we further obtain that

$$
\begin{aligned}
c & =\sum_{m=1}^{K} \sum_{n=1}^{K} \mathbf{V}(m, n)=\sum_{m=1}^{K} \sum_{n=1}^{K} \mathbf{T}(m, n) \\
& =\sum_{m=1}^{K} \sum_{n=1}^{K} \mathbf{C}(m, n) s_{m}^{*} s_{n} \\
& =f^{2} p_{0} .
\end{aligned}
$$

By substituting (73) into (71), we obtain

$$
\Lambda=\sqrt{\frac{p_{0}}{f^{2} p_{0}}}=\frac{1}{f} .
$$


In this case, with all dual variables equal to zero, each $\lambda_{k}$ is real and identical, which further leads to the expression of the precoded signal vector for $\mathrm{CI}$ as

$$
\begin{aligned}
\mathbf{x}_{C I} & =\mathbf{W} \mathbf{s}=\frac{1}{K} \cdot \mathbf{H}^{H}\left(\mathbf{H} \mathbf{H}^{H}\right)^{-1} \frac{1}{f} \mathbf{s} \hat{\mathbf{s}} \\
& =\frac{1}{f} \cdot \mathbf{H}^{H}\left(\mathbf{H} \mathbf{H}^{H}\right)^{-1} \mathbf{s} \\
& =\mathbf{x}_{Z F},
\end{aligned}
$$

which is identical to the precoded signal vector based on $\mathrm{ZF}$, where we denote $\mathbf{x}_{C I}$ as the transmit signal vector for the CI precoding.

The above results show that the conventional $\mathrm{ZF}$ precoding can be regarded as a special case of the CI precoding with all the dual variables being zero, as demonstrated in (68). The performance of ZF method is therefore the lower-bound of the CI precoding. We shall discuss under what conditions the $\mathrm{CI}$ precoding is equivalent to the $\mathrm{ZF}$ approach in the following section. It can be further observed that the performance of the CI precoding will be superior to the $\mathrm{ZF}$ scheme if not all the dual variables are zero, as shown in (67) where the existence of non-zero dual variables will increase the minimum value in $\boldsymbol{\Lambda}$. We further note that when the optimality is achieved, the minimum value in $\boldsymbol{\Lambda}$ is guaranteed to be not smaller than (74), for otherwise the $\mathrm{ZF}$ precoding will generate a larger minimum value in $\boldsymbol{\Lambda}$, which means that $\mathrm{ZF}$ should be the optimal and this causes contradiction.

\section{Proposed Iterative Closed-Form Scheme}

In this section, our proposed iterative close-form scheme is introduced. Throughout this section, we consider the case of strict phase rotation, while the extension to the nonstrict phase rotation is trivial and briefly included, as both optimization problems share the same problem formulation, discussed in Section IV. To introduce the proposed scheme, we first transform the expression of $\mathbf{u}$ in (63) into

$$
\mathbf{u}=\frac{1}{2} \mathbf{G q}+\frac{\mathbf{a}}{c}
$$

where $\mathbf{G}$ is defined as

$$
\mathbf{G}=\mathbf{V}-\mathbf{\Phi}
$$

Then, based on the optimality conditions in (56), as long as we find $\mathbf{a} \mathbf{u}$ and the corresponding dual vector $\mathbf{q}$ that satisfy (56), the obtained $\mathbf{u}$ is the optimal solution for $\mathcal{P}_{4}$. This further leads to the following optimization problem

$$
\begin{array}{ll}
\mathcal{P}_{9}: & \text { find } \mathbf{q} \\
\text { s.t. } & \mathbf{u}=\frac{1}{2} \mathbf{G q}+\frac{\mathbf{a}}{c} \\
& \mathbf{1}^{T} \mathbf{u}-1=0 \\
& \mu_{k} q_{k}=0, \mu_{k} \geq 0, q_{k} \geq 0, \forall k \in \mathcal{K}
\end{array}
$$

For clarity of description, we define a set $\mathcal{S}$ as

$$
\mathcal{S}=\left\{k \mid a_{k}<0, \forall k \in \mathcal{K}\right\} .
$$

In the following based on $\mathcal{S}$ we discuss the solution of $\mathcal{P}_{9}$ and propose the iterative closed-form scheme.

\section{A. $\mathcal{S}=\emptyset$}

When $\mathcal{S}=\emptyset$, this means $a_{k} \geq 0, \forall k \in \mathcal{K}$. Then, based on the fact that $\mathbf{1}^{T} \mathbf{a}=c$ in (61), it is obvious that

$$
\mathbf{u}=\frac{\mathbf{a}}{c}, q_{k}=0, \forall k \in \mathcal{K}
$$

satisfies all the conditions in (78). Therefore, when $\mathcal{S}=\emptyset$, the optimal $\mathbf{u}^{*}$ and $\mathbf{q}^{*}$ can be obtained as

$$
\mathbf{u}^{*}=\frac{\mathbf{a}}{c}, \mathbf{q}^{*}=\mathbf{0}
$$

In this case, based on our analysis in Section IV the CI precoding is identical to the $\mathrm{ZF}$ approach, where no performance gains can be obtained.

\section{B. $\operatorname{card}(\mathcal{S}) \neq \emptyset$}

When $\operatorname{card}(\mathcal{S}) \neq \emptyset$, this means that there is at least one entry in a that is smaller than zero. It is then obvious that the optimal $\mathbf{u}^{*} \neq \frac{\mathbf{a}}{c}$ due to the requirement that $\mu_{k} \geq 0$. In this case, we can obtain that not all the dual variables are zero, and we need to introduce at least one positive $q_{i}$ such that each $\mu_{k} \geq 0$. We firstly set

$$
\mathbf{u}=\frac{\mathbf{a}}{c}, \mathbf{q}=\mathbf{0} .
$$

Subsequently, we sort the entries in $\mathbf{u}$ following an ascending order, expressed as

$$
\mathbf{d}=\operatorname{sort}(\mathbf{u})
$$

where $\mathbf{d}$ is the sorted vector and $\mathbf{d}=\left[d_{1}, d_{2}, \cdots, d_{K}\right]^{T}$. sort $(\cdot)$ denotes the sort function, and without loss of generality we denote $k$ as the minimum value in $\mathbf{u}$, which leads to

$$
\mu_{k}=\min (\mathbf{u})=d_{1}
$$

With (84) we can also obtain $a_{k}<0$ and $d_{1}<0$. Let us firstly introduce only one positive dual variable $q_{k}$ that corresponds to $\mu_{k}$ while keeping other dual variables zero. Based on the complementary slackness condition, when $q_{k} \neq 0$, we obtain $\mu_{k}=0$, and this further leads to

$$
\begin{aligned}
& \mu_{k}=\frac{1}{2} \sum_{i=1}^{K} \mathbf{G}(k, i) q_{i}+\frac{a_{k}}{c}=0 \\
\Rightarrow & \frac{1}{2} \mathbf{G}(k, k) q_{k}+\frac{a_{k}}{c}=0 \\
\Rightarrow & q_{k}=-\frac{2 a_{k}}{\mathbf{G}(k, k) c} .
\end{aligned}
$$

where based on the definition of $\mathbf{G}$ we can verify that $\mathbf{G}(k, k)>0$. Based on the fact that $a_{k}<0$ it is then obtained that $q_{k}>0$. We further define a vector $\mathbf{i}$

$$
\mathbf{i}=[k], \forall q_{k} \neq 0,
$$

and a set $\mathcal{I}$ that consists of all the entries in $\mathbf{i}$, where we denote $\mathbf{i}=\left[i_{1}, i_{2} \cdots, i_{M}\right]$ and $\operatorname{card}(\mathcal{I})=M$. By updating $\mathbf{q}$ with the updated $q_{k}$ based on (85), the updated $\mathbf{u}$ can be expressed as

$$
\mathbf{u}=\frac{1}{2} \sum_{k \in \mathcal{I}} \mathbf{g}_{k} q_{k}+\frac{\mathbf{a}}{c},
$$


where we have decomposed $\mathbf{G}=\left[\mathbf{g}_{1}, \mathbf{g}_{2}, \cdots, \mathbf{g}_{K}\right]$, and (87) satisfies $\mu_{k}=0$. We verify whether the minimum value in the updated $\mathbf{u}$ satisfies the non-negative condition, and the updated $\mathbf{u}$ is the optimal solution of $\mathcal{P}_{9}$ if $\min (\mathbf{u})$ is non-negative. If this condition is not satisfied, this means that one dual variable is not enough and we need to introduce an additional dual variable. In this case, we first sort the updated $\mathbf{u}$ based on (83) and then find the minimum value in the updated $\mathbf{d}$, where without loss of generality we denote

$$
\mu_{l}=d_{1}, q_{l} \neq 0,
$$

where we note that $d_{1}$ in (88) is different from $d_{1}$ in (84) as $\mathbf{u}$ has been updated. With the existence of two non-zero dual variables, we obtain $\mathbf{i}=[k, l]$ and $\mathcal{I}=\{k, l\}$. We can then formulate a matrix $\mathbf{Z} \in \mathcal{C}^{\operatorname{card}(\mathcal{S}) \times \operatorname{card}(\mathcal{S})}$ as

$$
\mathbf{Z}=\left[\begin{array}{ll}
\mathbf{G}(k, k) & \mathbf{G}(k, l) \\
\mathbf{G}(l, k) & \mathbf{G}(l, l)
\end{array}\right] .
$$

By defining

$$
\tilde{\mathbf{q}}=\left[q_{k}, q_{l}\right]^{T}, \tilde{\mathbf{a}}=\left[a_{k}, a_{l}\right]^{T}, \tilde{\mathbf{u}}=\left[\mu_{k}, \mu_{l}\right]^{T}
$$

that consists of the entries that correspond to the numbers of non-zero dual variables, we obtain

$$
\tilde{\mathbf{u}}=\mathbf{0},
$$

which is due to the complementary slackness condition. With (87) and (91), we can further obtain $\tilde{\mathbf{q}}$ as

$$
\begin{aligned}
& \tilde{\mathbf{u}}=\frac{1}{2}\left(\left[\begin{array}{c}
\mathbf{G}(k, k) \\
\mathbf{G}(l, k)
\end{array}\right] q_{k}+\left[\begin{array}{c}
\mathbf{G}(k, l) \\
\mathbf{G}(l, l)
\end{array}\right] q_{l}\right)+\frac{\tilde{\mathbf{a}}}{c}=\mathbf{0} \\
\Rightarrow & \frac{1}{2} \mathbf{Z} \tilde{\mathbf{q}}+\frac{\tilde{\mathbf{a}}}{c}=\mathbf{0} \\
\Rightarrow & \tilde{\mathbf{q}}=-\frac{2}{c} \cdot \mathbf{Z}^{-1} \tilde{\mathbf{a}} .
\end{aligned}
$$

If each entry in the obtained $\tilde{\mathbf{q}}$ satisfies the non-negative condition, we update $\mathbf{u}$ based on (87) with the updated $\mathcal{I}$, and further check whether the minimum value in the updated $\mathbf{u}$ satisfies the non-negative condition.

Nevertheless, when $\operatorname{card}(\mathcal{S})>1$, it cannot be guaranteed that each entry in the obtained $\tilde{\mathbf{q}}$ in (92) satisfies the nonnegative condition. In this case, a retraction approach is required. To be more specific, if there is one entry in the obtained $\tilde{\mathbf{q}}$ that is negative, we firstly find the corresponding number of the negative dual variable, given by

$$
k=\text { find }\left(q_{k}<0\right),
$$

where the 'find' function returns the index of the negative entry in q. We further obtain the corresponding column index of $k$ in $\mathbf{i}$, expressed as

$$
i_{m}=k \text {. }
$$

We then reset

$$
\mathbf{i}=\left[i_{1}, i_{2}, \cdots, i_{m-1}\right],
$$

which means that there are currently $(m-1)$ positive dual variables and we set all the obtained dual variables obtained after $(m-1)$ to 0 . With (95), we reformulate the corresponding $\mathcal{I}$ and $\mathbf{u}$. Then, for the $m$-th dual variable, instead of selecting the number that corresponds to the minimum value in $\mathbf{d}$ as in (84), we select $\mu_{m}$ that corresponds to the second minimum value in $\mathbf{d}$. Based on (83) we obtain

$$
\mu_{m}=d_{2},
$$

and we update $\mathbf{i}$ and $\mathcal{I}$. With the updated $\mathcal{I}$, we calculate $\tilde{\mathbf{q}}$ based on (92), and we repeat the above process (83)-(96) by increasing the number of non-zero dual variables one at a time until all the entries in the updated $\mathbf{u}$ are non-negative, on the condition that in each step the entries in the obtained $\tilde{\mathbf{q}}$ are non-negative.

\section{The Iterative Algorithm}

Based on the above description, we summarize the proposed scheme for strict phase rotation in Algorithm 1. Since the algorithm will find the $\mathbf{u}$ and $\mathbf{q}$ that satisfy the KKT conditions for optimality, the obtained $\mathbf{u}$ is therefore the optimal solution to the optimization problem $\mathcal{P}_{4}$ for strict phase rotation, and the optimal precoding matrix can be obtained with (29)

\section{Algorithm 1 Proposed Iterative Closed-form Scheme for Strict} Phase Rotation

input : $\mathbf{s}, \mathbf{H}$

output : $\mathbf{W}^{*}$

Initialize $\mathbf{i}=[], \mathcal{I}=\emptyset, \mathbf{N}=[1], t=1$, and $n=0$;

Calculate $\mathbf{T}$ based on (20); Obtain $\mathbf{V}=\Re(\mathbf{T})$;

Calculate a based on (59) and $c$ based on (61);

Calculate $\mathbf{G}=\mathbf{V}-\frac{\mathbf{a a}^{T}}{c} ;$ Calculate $\mathbf{u}=\frac{\mathbf{a}}{c}$;

Obtain $\mathcal{S}$ based on (79);

if $\operatorname{card}(\mathcal{S})=\emptyset$ then

Obtain $\mathbf{u}^{*}=\mathbf{u}$;

else

while $\min (\mathbf{u})<0$ and $n<n_{\max }$ do

$\mathbf{d}=\operatorname{sort}(\mathbf{u})$;

find $k$ such that $\mu_{k}=d_{t}$;

Stack $\mathbf{N}=\left[\begin{array}{cc}\mathbf{N} & 1\end{array}\right]$;

Update $\mathbf{i}$ and $\mathcal{I}$; Formulate $\mathbf{Z}$ based on $\mathcal{I}$ and $\mathbf{G}$;

Calculate $\tilde{\mathbf{q}}$ based on $\mathcal{I}$ and $\mathbf{Z}$ with (92);

if $\min (\tilde{\mathbf{q}}) \geq 0$ then

Update $\mathbf{u}$ based on (87);

$t=1$;

else

find $k$ such that $q_{k}=\min (\tilde{\mathbf{q}})$;

find $m$ such that $i_{m}=k$;

Set i based on (95); Update $\mathcal{I}$;

Formulate $\mathbf{Z}$ based on $\mathcal{I}$ and $\mathbf{G}$;

Update $\tilde{\mathbf{q}}$ with (92); Update $\mathbf{u}$ with (86);

Reformulate $\mathbf{N}=\mathbf{N}(1: m)$;

Update $\mathbf{N}(m) \leftarrow \mathbf{N}(m)+1$;

Update $t=\mathbf{N}(m)$;

\section{end if}

$n \leftarrow n+1$;

end while

Obtain $\mathbf{u}^{*}=\mathbf{u}$;

end if

Calculate $\mathbf{W}^{*}$ based on the obtained $\mathbf{u}^{*}$ with (30). 


$$
\begin{aligned}
\mathrm{C}_{\mathcal{P}_{10}}= & (K+2)^{0.5}\left(N_{t}+1\right)\left[2 N_{t}^{2}+2 N_{t}+K+2\right] . \\
& \ln \left(\frac{\left(N_{t}+K+1\right)\left(N_{t}+2\right)+4 N_{t}+3 K+8+\sum_{k=1}^{K} \sum_{i=1}^{N_{t}}\left|\frac{s_{i}}{s_{k}} \mathbf{h}_{k}(i)\right|+\sqrt{\frac{p_{0}}{K^{2}}}+\varepsilon^{2}}{\varepsilon}\right)
\end{aligned}
$$

accordingly. While the algorithm is for the case of the strict phase rotation, it is trivial to extend to the case of non-strict phase rotation by substituting $\mathbf{V}$ with $\hat{\mathbf{V}}$ in (48) to obtain the optimal $\hat{\mathbf{u}}^{*}$. Subsequently, the optimal precoding matrix for non-strict phase rotation is obtained with (50).

We note that, while the above algorithm includes an iterative design, within each iteration a closed-form solution is indeed obtained and the algorithm only includes linear matrix manipulations, which is computationally efficient. Moreover, it will be shown that the number of iterations required is small, especially when the number of users is small. We further note that, while the KKT optimality conditions are not satisfied before the final iteration, the solution obtained within each iteration is indeed a feasible solution that satisfies the power constraint for the precoding and achieves an improved performance over ZF but a sub-optimal performance compared to the optimal CI precoding. Indeed, the obtained precoder in each iteration approaches the optimal precoding strategy with the increasing iteration number. Therefore, the proposed iterative scheme can also achieve a flexible performance-complexity tradeoff by limiting the maximum number of iterations $n_{\max }$ in Algorithm 1.

\section{COMPutational COMPlEXity ANALysis}

In this section, we study the computational costs of the optimization-based CI precoding and the proposed iterative closed-form algorithm. For the optimization-based approaches, the computational cost is evaluated based on the arithmetic time complexity bound [43], and the complexity of the proposed algorithm is evaluated in terms of the required number of real multiplications and additions [44].

\section{A. Optimization-based CI Precoding}

Without loss of generality, we focus on the CI precoding for strict phase rotation for the optimization-based approaches, while the extension to the case of non-strict phase rotation is trivial, as they share a similar problem formulation.

For the optimization-based CI precoding in the case of strict phase rotation, the complexity is dominated by solving the convex optimization problem $\mathcal{P}_{2}$ via the interior-point method. For $\mathcal{P}_{2}$, based on the fact each $\mathbf{w}_{i} s_{i}$ is identical, we can transform $\mathcal{P}_{2}$ into an optimization on $\mathbf{w}_{i}$ only, and we express it in a standard SOCP form as

$$
\begin{array}{ll}
\mathcal{P}_{10}: & \min _{\mathbf{x}} \mathbf{c}^{T} \mathbf{x} \\
\text { s.t. } & \mathbf{c}_{k}^{T} \mathbf{x} \leq 0, \forall k \in \mathcal{K} \\
& \|\mathbf{A x}\|_{2} \leq \sqrt{\frac{p_{0}}{K^{2}}}
\end{array}
$$

where we introduce $\mathbf{x}=\left[\mathbf{w}_{i}^{T}, t\right]^{T} \in \mathcal{C}^{\left(N_{t}+1\right) \times 1}, \mathbf{c}=$ $[0, \cdots, 0,-1]^{T} \in \mathcal{C}^{\left(N_{t}+1\right) \times 1}, \mathbf{c}_{k}=\left[\left(-\frac{K s_{i}}{s_{k}} \cdot \mathbf{h}_{k}\right)^{T}, 1\right]^{T} \in$ $\mathcal{C}^{\left(N_{t}+1\right) \times 1}$, and $\mathbf{A}=\left[s_{i} \cdot \mathbf{I}, \mathbf{0}\right] \in \mathcal{C}^{N_{t} \times\left(N_{t}+1\right)}$. Compared to $\mathcal{P}_{2}$, we note that the constraints on $\lambda_{k}$ has been included in each $\mathbf{c}_{k}$ implicitly. Subsequently, based on [43] we can obtain the arithmetic time complexity bound of the above optimization via the interior-point methods, given by

$$
\mathrm{C}_{\mathcal{P}_{10}}=(M+1)^{0.5} N\left(N^{2}+M+L^{2}\right) \cdot \mathrm{D}(\mathbf{p}, \varepsilon),
$$

where $\varepsilon$ is the target accuracy of the solution, $N$ is the dimension of the variable $\mathbf{x}, M$ is the total number of constraints, and $L$ is the dimension of $\mathbf{A x}$ in the SOC constraint. Based on the construction of $\mathcal{P}_{10}$, we obtain

$$
M=K+1, \quad N=N_{t}+1, L=N_{t},
$$

which further leads to the expression of $\mathrm{C}_{\mathcal{P}_{10}}$ as

$$
\begin{aligned}
& \mathrm{C}_{\mathcal{P}_{10}} \\
= & (K+2)^{0.5}\left(N_{t}+1\right)\left[\left(N_{t}+1\right)^{2}+K+1+N_{t}^{2}\right] \cdot \mathrm{D}(\mathbf{p}, \varepsilon) \\
= & (K+2)^{0.5}\left(N_{t}+1\right)\left[2 N_{t}^{2}+2 N_{t}+K+2\right] \cdot \mathrm{D}(\mathbf{p}, \varepsilon) .
\end{aligned}
$$

$\mathrm{D}(\mathbf{p}, \varepsilon)$ is the number of digits of accuracy for a solution with the target accuracy $\varepsilon$, and is given by

$$
\mathrm{D}(\mathbf{p}, \varepsilon)=\ln \left(\frac{\operatorname{Dim}(\mathbf{p})+\|\mathbf{p}\|_{1}+\varepsilon^{2}}{\varepsilon}\right),
$$

where the column vector $\mathbf{p}$ represents a permutation vector that contains the parameters in both the objective function and the constraints. For the constructed problem $\mathcal{P}_{10}, \mathbf{p}$ is given as

$\mathbf{p}=\left[(K+1),\left(N_{t}+1\right), N_{t},-1, \mathbf{c}_{1}^{T}, \cdots, \mathbf{c}_{K}^{T}, s_{i} \mathbf{1}^{T}, \sqrt{\frac{p_{0}}{K^{2}}}\right]^{T}$.

In (101), Dim (p) denotes the dimension of the permutation vector $\mathbf{p}$, and is accordingly obtained as

$$
\begin{aligned}
\operatorname{Dim}(\mathbf{p}) & =(M+L)(N+1)+M+N+3 \\
& =\left(N_{t}+K+1\right)\left(N_{t}+2\right)+N_{t}+K+5 .
\end{aligned}
$$

Based on the expression of $\mathbf{p}$, we further obtain $\|\mathbf{p}\|_{1}$ as

$$
\|\mathbf{p}\|_{1}=3 N_{t}+2 K+3+K \sum_{k=1}^{K} \sum_{i=1}^{N_{t}}\left|\frac{s_{i}}{s_{k}} \mathbf{h}_{k}(i)\right|+\sqrt{\frac{p_{0}}{K^{2}}} .
$$

Given the expression of $\operatorname{Dim}(\mathbf{p})$ and $\|\mathbf{p}\|_{1}$, we arrive at the final expression of the arithmetic complexity of $\mathcal{P}_{10}$, which is given by (105) at the topic of this page. The analytical time complexity bound of $\mathcal{P}_{4}$ can be similarly obtained by transforming the QP formulation of $\mathcal{P}_{4}$ into a SOCP formulation, and is omitted for brevity. 


\section{B. Iterative Closed-Form Algorithm}

For the proposed iterative closed-form algorithm in Section $\mathrm{V}$, the complexity is evaluated by the required number of real multiplications and additions in matrix operation. Specifically, we observe that its complexity is dominated by the matrix calculation in (92), and the size of $\mathbf{Z}^{-1}$ and $\tilde{\mathbf{a}}$ is dependent on the current number of iteration ' $n$ ', i.e., $\mathbf{Z}^{-1} \in \mathcal{R}^{(n+1) \times(n+1)}$ and $\tilde{\mathbf{a}} \in \mathcal{R}^{(n+1) \times 1}$. Following [44], we obtain that the calculation of $\mathbf{Z}^{-1} \tilde{\mathbf{a}}$ requires $(n+1)^{2}$ multiplications and $n(n+1)$ additions. Accordingly, by assuming a maximum number of iteration $n_{\max }$, the complexity of the proposed iterative closed-form algorithm is approximately obtained in terms of the required number of real operations as

$\mathrm{C}_{\mathrm{Alg} 1}=\sum_{n=0}^{n_{\max }}\left[(n+1)^{2}+n(n+1)\right]=\sum_{n=0}^{n_{\max }}\left[2 n^{2}+3 n+1\right]$.

Nevertheless, we note that the above two expressions for the evaluation of complexity may not be directly comparable, as the complexity of the optimization problem is evaluated based on the time complexity bound, while the complexity of the proposed algorithm is evaluated based on the required number of real operations.

\section{NUMERICAL RESUlTS}

In this section, the numerical results of the proposed schemes are presented and compared with the traditional CI precoding based on the Monte Carlo simulations. In each plot, we assume the total transmit power available as $p_{0}=1$, and the transmit SNR per antenna as $\rho=1 / \sigma^{2}$. We compare our proposed iterative schemes with the traditional closedform ZF-based methods, optimization-based SINR balancing approaches [7][10], and CI precoding approaches $\mathcal{P}_{1}$ and $\mathcal{P}_{5}$ for both strict and non-strict phase rotation. Both the uncoded and coded BER results are presented.

For clarity the following abbreviations are used throughout this section:

1) ' $\mathrm{ZF}$ ': traditional $\mathrm{ZF}$ scheme with symbol-level power normalization in (52) and (53);

2) 'RZF': traditional RZF scheme with symbol-level power normalization, where the precoded signal vector is given by

$$
\mathbf{x}_{R Z F}=\mathbf{W}_{R Z F} \mathbf{s}=\frac{1}{f} \cdot \mathbf{H}^{H}\left(\mathbf{H H}^{H}+\frac{K}{\rho} \cdot \mathbf{I}\right)^{-1} \mathbf{s}
$$

with the symbol-level scaling factor $f$ given by

$$
f=\frac{\left\|\mathbf{W}_{R Z F} \mathbf{s}\right\|_{2}}{\sqrt{p_{0}}}
$$

3) 'SINR Balancing': the SINR balancing approach based on bisection search method [7];

4) 'CI-opt, Strict/Non-Strict': traditional CI precoding, $\mathcal{P}_{1}$ for strict phase rotation and $\mathcal{P}_{5}$ for non-strict phase rotation;

5) 'CI-CF, Strict/Non-Strict': the proposed iterative closedform scheme for strict/non-strict phase rotation based on Algorithm 1.

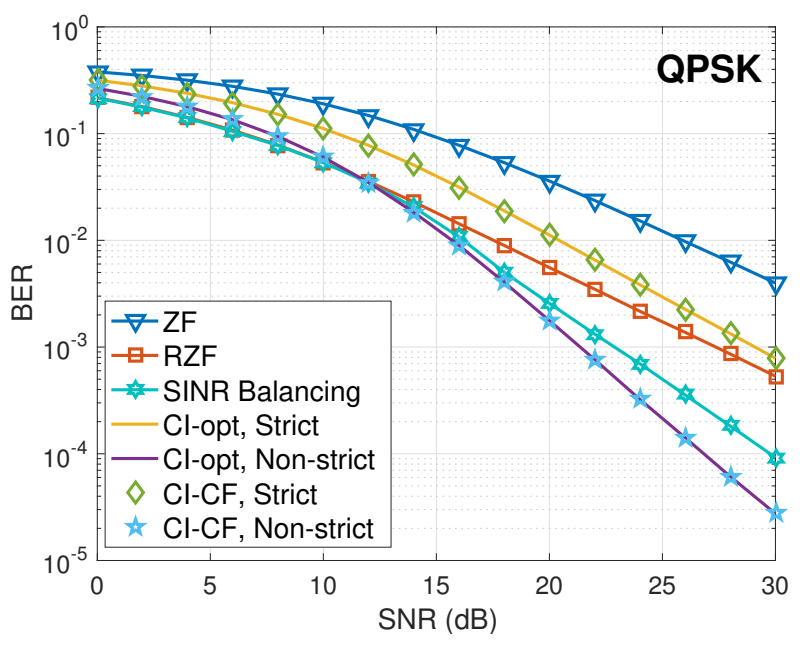

Fig. 4: Uncoded BER v.s. transmit SNR, QPSK, $N_{t}=K=8$

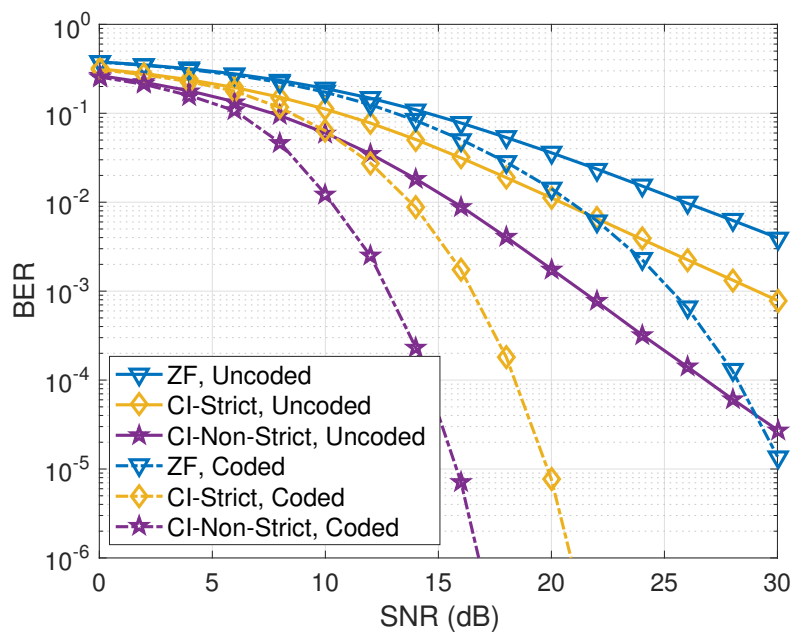

Fig. 5: Coded BER v.s. transmit SNR, QPSK, $N_{t}=K=8$, LDPC with code rate $5 / 6$

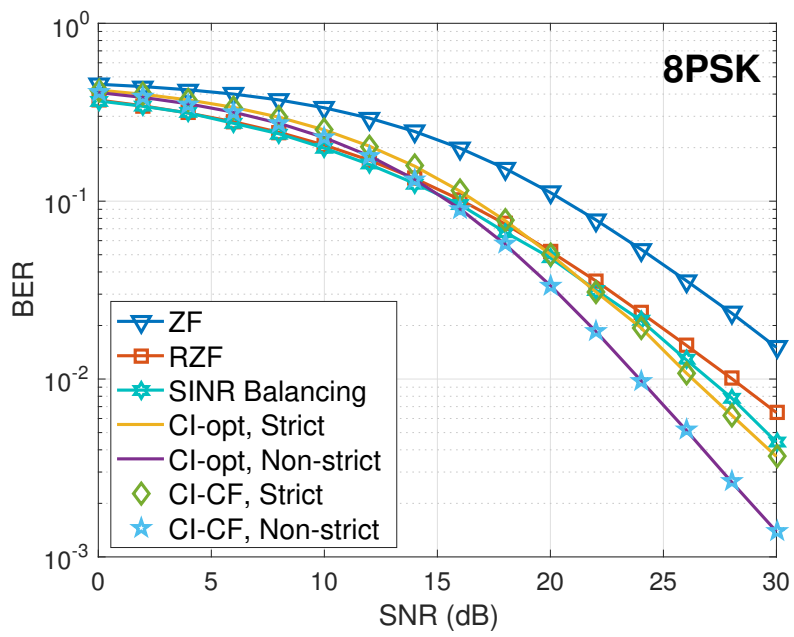

Fig. 6: Uncoded BER v.s. transmit SNR, 8PSK, $N_{t}=K=8$ 


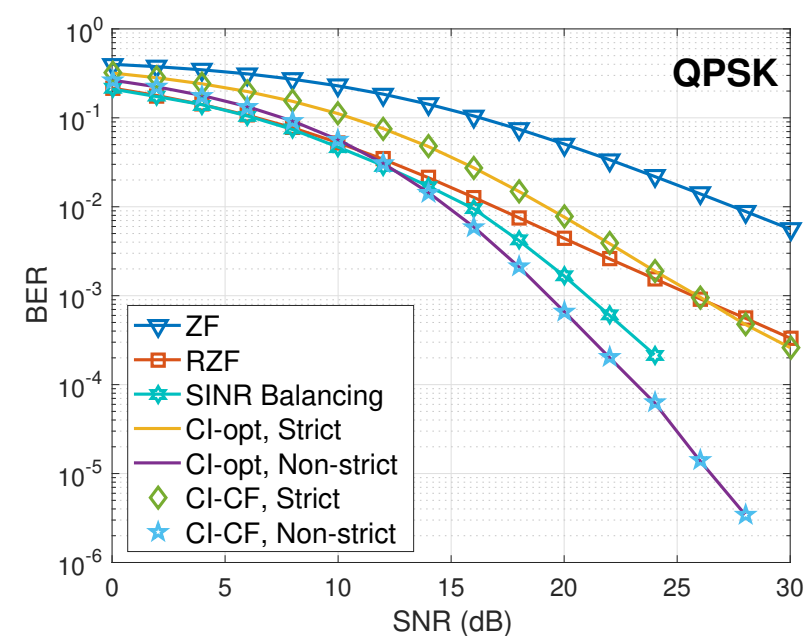

Fig. 7: Uncoded BER v.s. transmit SNR, QPSK, $N_{t}=K=12$

In Fig. 4, we compare the bit error rate (BER) performance of different schemes with QPSK modulation, and $N_{t}=K=$ 8. As can be observed, the CI precoding approaches for both strict phase rotation and non-strict phase rotation achieve an improved performance over the ZF approach, and the gain for non-strict phase rotation is more significant. For the CI precoding for non-strict phase rotation at high SNR regime, we observe a SNR gain of more than $10 \mathrm{~dB}$ over $\mathrm{ZF}$ and 8dB SNR gain over RZF. Moreover, we observe that the proposed iterative closed-form algorithm achieves exactly the same performance as the optimization-based CI precoding, which validates the effectiveness of the proposed method in Section V. In Fig. 5, we further present the coded BER result for the same system setup, where we employ the low-density parity-check (LDPC) coding with the code rate 5/6. With channel coding, all precoding methods achieve a significantly improved performance compared to the case without channel coding, and the performance gains of the CI-based symbollevel precoding method persist in the presence of channel coding.

In Fig. 6, we show the BER performance with respect to the increasing transmit SNR when 8PSK modulation is employed, where $N_{t}=K=8$. Similarly, both transmit precoding approaches based on the $\mathrm{CI}$ achieve an improved performance over the ZF method, and the proposed iterative closed-form schemes achieve the same performance as the optimizationbased schemes. At high SNR $(\rho>20 \mathrm{~dB})$, both CI-based approaches outperform the ZF-based schemes. For CI with non-strict phase rotation, we observe a SNR gain of over $7 \mathrm{~dB}$ compared to ZF, and a SNR gain of 5dB compared to RZF precoding.

Fig. 7 shows the BER performance of different schemes for QPSK with $N_{t}=K=12$, where a similar BER trend can be observed, where the CI-based precoding with nonstrict phase rotation achieves a significant better performance than other precoding approaches. Particularly, comparing Fig. 4 and Fig. 7, we observe that the performance gains of the CI-based approaches over the conventional ZF precoding are

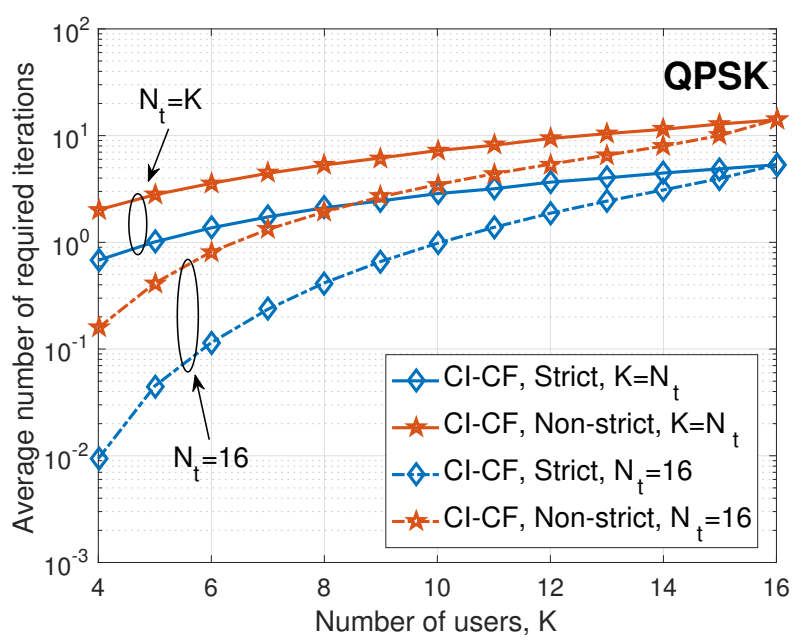

Fig. 8: Average number of iterations required for the iterative closed-form scheme, QPSK, $N_{t}=K$ and $N_{t}=16$

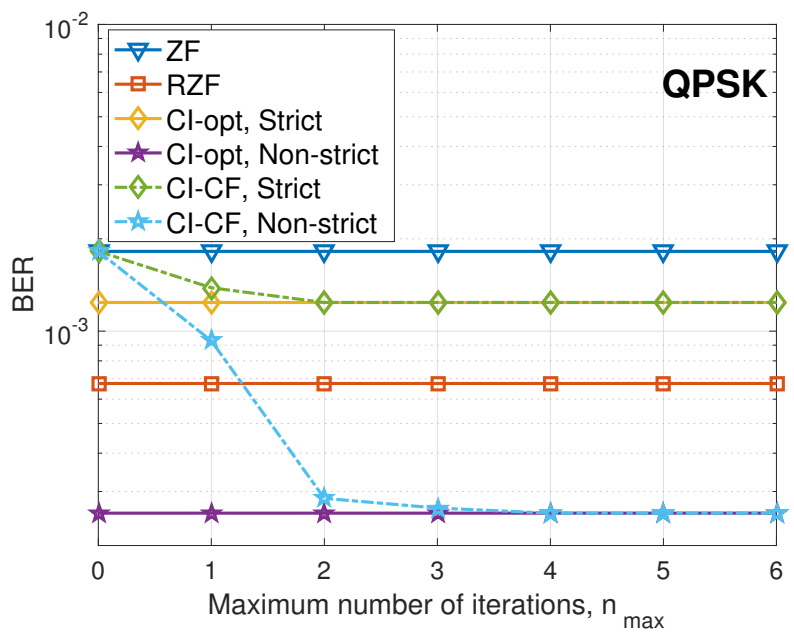

Fig. 9: Uncoded BER v.s. maximum number of iterations $n_{\max }$, QPSK, $N_{t}=K=4$, SNR $=30 \mathrm{~dB}$

more significant with the increase in the number of antennas and users.

In Fig. 8, the average number of iterations required for the proposed iterative scheme is numerically studied with the increasing number of users, where we include two cases of $N_{t}=K$ and $N_{t}=16$. Generally, we observe that the average number of iterations increases with the increase in the number of users, as a larger number of users means a high possibility that more entries in a can be negative. Moreover, we observe that the number of required iterations is smaller when the number of users is smaller than that of transmit antennas. The non-strict phase rotation requires more iterations than the strict phase rotation because the problem size is doubled. We also observe that when the number of users $K$ is small, the average number of iterations can be smaller than 1 because the number of iterations is zero when $\mathcal{S}=\emptyset$.

To show the flexible performance-complexity tradeoff for the proposed algorithm, in Fig. 9 we depict the BER per- 


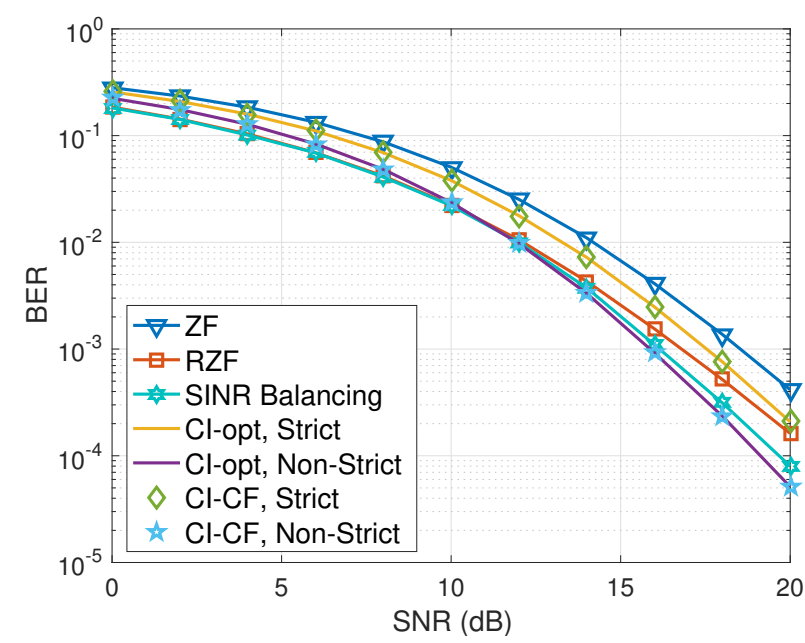

Fig. 10: Uncoded BER v.s. transmit SNR, QPSK, $K=8$, $N_{t}=10$

formance of the proposed iterative approach with respect to the maximum iteration number $n_{\max }$, where $N_{t}=K=4$. As expected, we observe that the performance of the iterative approach is identical to the conventional $\mathrm{ZF}$ approach when $n_{\max }=0$. With $n_{\max }$ increases, the performance of the iterative method approaches the optimal CI-based precoding, which validates our statement in Section V-C.

In Fig. 10, we further compare the uncoded BER result for the case of $N_{t}>K$, where we consider a total number of $K=8$ users served by a BS with $N_{t}=10$ transmit antennas. We observe a similar result compared to the case of $N_{t}=K$, where both CI-based precoding methods achieve an improved performance over the ZF precoding, while the CI-based precoding with non-strict phase rotation achieves the best BER result, and the performance gain is more prominent in the high SNR regime.

As it is difficult to provide a generic framework to evaluate the analytical complexity for both the optimization-based CI precoding and the iterative closed-form CI precoding, in Fig. 11 we compare the execution time required for each scheme as an indication to show the potential complexity benefits of the proposed iterative closed-form scheme, where 'CIQP, Strict/Non-Strict' refers to the QP optimizations $\mathcal{P}_{4}$ and $\mathcal{P}_{8}$, and 'CI-SOCP, Strict/Non-Strict' refers to the SOCP optimizations $\mathcal{P}_{1}$ and $\mathcal{P}_{5}$. It is observed that the optimization for non-strict phase rotation requires more time to obtain the optimal solution that the strict-phase rotation because of the larger problem size, which is also clearly demonstrated in the literature [42]. It is also observed that solving the equivalent QP optimization is much faster than solving the original SOCP optimization. More importantly, our proposed iterative scheme is more time-efficient than the $\mathrm{QP}$ algorithms, which motivates the use of the symbol-level CI beamforming in practice.

\section{CONCLUSION}

In this paper, we study the symbol-level downlink precoding schemes based on CI, where both the strict and non-strict

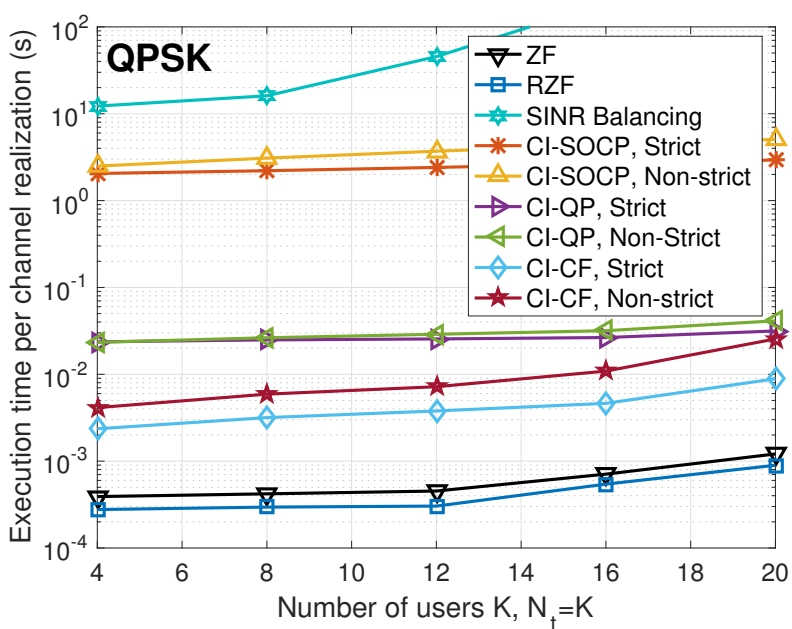

Fig. 11: Execution time required for different schemes, QPSK, $N_{t}=K, 5000$ channel realizations

phase rotation cases are considered. By analyzing the optimization problems with Lagrangian and KKT conditions, we firstly obtain the optimal structure of the precoding matrix, and further transform the optimization into a QP over a simplex by formulating the dual problem. We show that the optimizations for strict and non-strict phase rotation are equivalent in terms of the problem formulation. We further illustrate that ZF precoding is a special case and lower-bound of the CI precoding. The proposed iterative closed-form scheme is shown to achieve an identical performance to the optimization-based schemes with a reduced computational cost, which enables the use of symbol-level CI precoding in practical wireless systems. Our future work is to consider the possibility of closed-form CI precoding methods for QAM modulations.

\section{REFERENCES}

[1] L. Zheng and D. N. C. Tse, "Diversity and Multiplexing: A Fundamental Tradeoff in Multiple-Antenna Channels," IEEE Trans. Inf. Theory, vol. 49, no. 5, pp. 1073-1096, May 2003.

[2] M. Costa, "Writing on Dirty Paper," IEEE Trans. Inf. Theory, vol. IT-29, no. 3, pp. 439-441, May 1983.

[3] L. Sun and M. Lei, "Quantized CSI-based Tomlinson-Harashima Precoding in Multiuser MIMO Systems," IEEE Trans. Wireless Commun., vol. 12, no. 3, pp. 1118-1126, Mar. 2013.

[4] B. M. Hochwald, C. B. Peel, and A. L. Swindlehurst, "A VectorPerturbation Technique for Near-Capacity Multiantenna Multiuser Communication-part II: Perturbation," IEEE Trans. Commun., vol. 53, no. 3, pp. 537-544, Mar. 2005.

[5] T. Haustein, C. von Helmolt, E. Jorswieck, V. Jungnickel, and V. Pohl, "Performance of MIMO Systems with Channel Inversion," in Vehicular Technology Conference. IEEE 55th Vehicular Technology Conference. VTC Spring 2002 (Cat. No.02CH37367), vol. 1, 2002, pp. 35-39.

[6] C. B. Peel, B. M. Hochwald, and A. L. Swindlehurst, "A VectorPerturbation Technique for Near-Capacity Multiantenna Multiuser Communication-part I: Channel Inversion and Regularization,” IEEE Trans. Commun., vol. 53, no. 1, pp. 195-202, Jan. 2005.

[7] A. Wiesel, Y. C. Eldar, and S. Shamai (Shitz), "Linear Precoding via Conic Optimization for Fixed MIMO Receivers," IEEE Trans. Sig. Process., vol. 54, no. 1, pp. 161-176, Jan. 2006.

[8] M. F. Hanif, L.-N. Tran, A. Tolli, and M. Juntti, "Computationally Efficient Robust Beamforming for SINR Balancing in Multicell Downlink with Applications to Large Antenna Array Systems," IEEE Trans. Commun., vol. 62, no. 6, pp. 1908-1920, June 2014. 
[9] F. Wang, X. Wang, and Y. Zhu, "Transmit Beamforming for Multiuser Downlink with Per-Antenna Power Constraints," in 2014 IEEE International Conference on Communications (ICC), Sydney, NSW, 2014, pp. $4692-4697$.

[10] M. Schubert and H. Boche, "Solution of the Multiuser Downlink Beamforming Problem with Individual SINR Constraints," IEEE Trans. Veh. Tech., vol. 53, no. 1, pp. 18-28, Jan. 2004.

[11] M. Bengtsson and B. Ottersten, "Optimal and Suboptimal Transmit Beamforming," Handbook of Antennas in Wireless Communications, Jan. 2001.

[12] N. D. Sidiropoulos, T. N. Davidson, and Z.-Q. Luo, "Transmit Beamforming for Physical-Layer Multicasting," IEEE Trans. Sig. Process., vol. 54, no. 6, pp. 2239-2251, June 2006.

[13] E. Karipidis, N. D. Sidiropoulos, and Z.-Q. Luo, "Quality of Service and Max-Min Fair Transmit Beamforming to Multiple Cochannel Multicast Groups," IEEE Trans. Sig. Process., vol. 56, no. 3, pp. 1268-1279, Mar. 2008.

[14] C. Masouros, T. Ratnarajah, M. Sellathurai, C. B. Papadias, and A. K Shukla, "Known Interference in the Cellular Downlink: A Performance Limiting Factor or a Source of Green Signal Power?" IEEE Commun. Mag., vol. 51, no. 10, pp. 162-171, Oct. 2013.

[15] G. Zheng, I. Krikidis, C. Masouros, S. Timotheou, D. A. Toumpakaris, and Z. Ding, "Rethinking the Role of Interference in Wireless Networks," IEEE Commun. Mag., vol. 52, no. 11, pp. 152-158, Nov. 2014

[16] C. Masouros and E. Alsusa, "A Novel Transmitter-Based SelectivePrecoding Technique for DS/CDMA Systems," IEEE Sig. Process. Lett., vol. 14, no. 9, pp. 637-640, Sept. 2007.

[17] _ _ "Dynamic Linear Precoding for the Exploitation of Known Interference in MIMO Broadcast Systems," IEEE Trans. Wireless Commun. vol. 8, no. 3, pp. 1396-1401, Mar. 2009

[18] C. Masouros, "Correlation Rotation Linear Precoding for MIMO Broadcast Communicaitons," IEEE Trans. Sig. Process., vol. 59, no. 1, pp. 252-262, Jan. 2011.

[19] C. Masouros and G. Zheng, "Exploiting Known Interference as Green Signal Power for Downlink Beamforming Optimization," IEEE Trans. Sig. Process., vol. 63, no. 14, pp. 3628-3640, July 2015.

[20] M. Alodeh, S. Chatzinotas, and B. Ottersten, "Constructive Multiuser Interference in Symbol Level Precoding for the MISO Downlink Channel," IEEE Trans. Sig. Process., vol. 63, no. 9, pp. 2239-2252, May 2015.

[21] — , "Energy-Efficient Symbol-Level Precoding in Multiuser MISO based on Relaxed Detection Region," IEEE Trans. Wireless Commun., vol. 15 , no. 5, pp. 3755-3767, May 2016.

[22] C. Masouros, M. Sellathurai, and T. Ratnarajah, "Vector Perturbation based on Symbol Scaling for Limited Feedback MISO Downlinks," IEEE Trans. Sig. Process., vol. 62, no. 3, pp. 562-571, Feb. 2014.

[23] M. Alodeh, S. Chatzinotas, and B. Ottersten, "Symbol-Level Multiuser MISO Precoding for Multi-Level Adaptive Modulation," IEEE Trans. Wireless Commun., vol. 16, no. 8, pp. 5511-5524, Aug. 2017.

[24] C. Masouros and T. Ratnarajah, "Interference as a Source of Green Signal Power in Cognitive Relay Assisted Co-Existing MIMO Wireless Transmissions," IEEE Trans. Commun., vol. 60, no. 2, pp. 525-536, Feb. 2012.

[25] K. L. Law, C. Masouros, and M. Pesavento, "Transmit Precoding for Interference Exploitation in the Underlay Cognitive Radio Z-Channel," IEEE Trans. Sig. Process., vol. 65, no. 14, pp. 3617-3631, July 2017.

[26] A. Li and C. Masouros, "Hybrid Massive MIMO Unlicensed Transmission with 1-Bit Quantization," in 2017 IEEE Globecom Workshops (GC Wkshps), Singapore, 2017.

[27] — , "Constructive Interference Beamforming for Cooperative DualHop MIMO Relay Systems," in 2018 IEEE 87th Vehicular Technology Conference (VTC-Spring), Porto, Portugal, 2018.

[28] _ " "A Two-Stage Vector Perturbation Scheme for Adaptive Modulation in Downlink MU-MIMO," IEEE Trans. Veh. Tech., vol. 65, no. 9, pp. 7785-7791, Sept. 2016.

[29] F. Liu, C. Masouros, A. Li, T. Ratnarajah, and J. Zhou, "Interference Exploitation for Radar and Cellular Coexistence: The Power-Efficient Approach," IEEE Trans. Sig. Process., vol. 66, no. 14, pp. 3681-3695, July 2018.

[30] S. Timotheou, G. Zheng, C. Masouros, and I. Krikidis, "Exploiting Constructive Interference for Simultaneous Wireless Information and Power Transfer in Multiuser Downlink Systems," IEEE J. Sel. Areas Commun., vol. 34, no. 5, pp. 1772-1784, May 2016.

[31] A. Li and C. Masouros, "Exploiting Constructive Mutual Coupling in P2P MIMO by Analog-Digital Phase Alignment," IEEE Trans. Wireless Commun., vol. 16, no. 3, pp. 1948-1962, Mar. 2017.
[32] D. Spano, M. Alodeh, S. Chatzinotas, and B. Ottersten, "Symbol-Level Precoding for the Nonlinear Multiuser MISO Downlink Channel," IEEE Trans. Sig. Process., vol. 66, no. 5, pp. 1331-1345, March 2018.

[33] M. Alodeh, D. Spano, A. Kalantari, C. Tsinos, D. Christopoulos, S. Chatzinotas, and B. Ottersten, "Symbol-Level and Multicast Precoding for Multiuser Multiantenna Downlink: A Survey, Classification and Challenges," arXiv preprint, available online: https://arxiv.org/abs/1703.03617, 2018.

[34] A. Kalantari, C. Tsinos, M. Soltanalian, S. Chatzinotas, W. K. Ma, and B. Ottersten, "MIMO Directional Modulation M-QAM Precoding for Transceivers Performance Enhancement," in 2017 IEEE 18th International Workshop on Signal Processing Advances in Wireless Communications (SPAWC), Sapporo, 2017.

[35] _ "Spatial Peak Power Minimization for Relaxed Phase M-PSK MIMO Directional Modulation Transmitter," in 2017 25th European Signal Processing Conference (EUSIPCO), Kos, 2017.

[36] P. V. Amadori and C. Masouros, "Constant Envelope Precoding by Interference Exploitation in Phase Shift Keying-Modulated Multiuser Transmission," IEEE Trans. Wireless Commun., vol. 16, no. 1, pp. 538550, Jan. 2017.

[37] A. Li, C. Masouros, F. Liu, and A. L. Swindlehurst, "Massive MIMO 1Bit DAC Transmission: A Low-Complexity Symbol Scaling Approach," arXiv preprint, available online: https://arxiv.org/abs/1709.08278, 2018.

[38] A. Li, C. Masouros, and F. Liu, "Hybrid Analog-Digital 1-Bit DAC Transmission: From MMSE to Interference Exploitation," in 2018 26th European Signal Processing Conference (EUSIPCO), Rome, Italy, in press.

[39] L. Vandenberghe and S. Boyd, Convex Optimization. Cambridge University Press, 2004

[40] P. Wolfe, "The Simplex Method for Quadratic Programming," Econometrica, vol. 27, no. 3, pp. 382-398, July 1959.

[41] G. Cornuejols and R. Tutuncu, Optimization Methods in Finance. Cambridge University Press, Dec. 2006.

[42] F. Alizadeh and D. Goldfarb, "Second-Order Cone Programming," Mathematical Programming, vol. 95, no. 1, pp. 3-51, 2003.

[43] A. Ben-Tal and A. Nemirovski, Lectures on Modern Convex Optimization: Analysis, Algorithms, and Engineering Applications. Philadelphia, PA, USA: Society for Industrial and Applied Mathematics, 2001.

[44] R. Hunger, Floating Point Operations in Matrix-Vector Calculus. Munich University of Technology, Inst. for Circuit Theory and Signal Processing, 2005

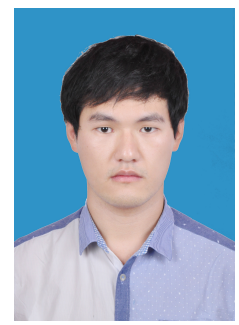

Ang Li (S'14-M'18) received his Ph.D. degree in the Communications and Information Systems research group, Department of Electrical and Electronic Engineering, University College London in 2018 . He is now a postdoctoral research associate in the School of Electrical and Information Engineering, University of Sydney. His research interests lie in the field of beamforming and signal processing techniques for MIMO systems. 


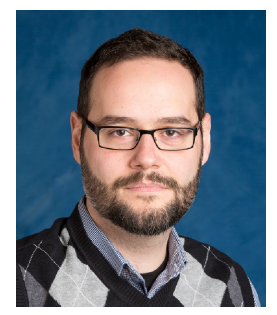

Christos Masouros (M'06-SM'14) received the Diploma degree in Electrical and Computer Engineering from the University of Patras, Greece, in 2004, and MSc by research and $\mathrm{PhD}$ in Electrical and Electronic Engineering from the University of Manchester, UK in 2006 and 2009 respectively. In 2008 he was a research intern at Philips Research Labs, UK. Between 2009-2010 he was a Research Associate in the University of Manchester and between 2010-2012 a Research Fellow in Queen's University Belfast. He has held a Royal Academy of Engineering Research Fellowship between 2011-2016.

$\mathrm{He}$ is currently a Senior Lecturer in the Communications and Information Systems research group, Dept. Electrical \& Electronic Engineering, University College London. His research interests lie in the field of wireless communications and signal processing with particular focus on Green Communications, Large Scale Antenna Systems, Cognitive Radio, interference mitigation techniques for MIMO and multicarrier communications. He was the recipient of the Best Paper Award in the IEEE GlobeCom conference 2015, and has been recognised as an Exemplary Editor for the IEEE Communications Letters, and as an Exemplary Reviewer for the IEEE Transactions on Communications. He is an Associate Editor for IEEE Communications Letters, and Guest Editor for IEEE Journal on Selected Topics in Signal Processing issue "Exploiting Interference towards Energy Efficient and Secure Wireless Communications". 